\title{
Human cortical connectome reconstruction from diffusion weighted MRI: The effect of tractography algorithm
}

Citation for published version (APA):

Bastiani, M., Shah, N. J., Goebel, R., \& Roebroeck, A. (2012). Human cortical connectome reconstruction from diffusion weighted MRI: The effect of tractography algorithm. Neuroimage, 62(3), 1732-1749. https://doi.org/10.1016/j.neuroimage.2012.06.002

Document status and date:

Published: 01/09/2012

DOI:

10.1016/j.neuroimage.2012.06.002

Document Version:

Publisher's PDF, also known as Version of record

Document license:

Taverne

Please check the document version of this publication:

- A submitted manuscript is the version of the article upon submission and before peer-review. There can be important differences between the submitted version and the official published version of record.

People interested in the research are advised to contact the author for the final version of the publication, or visit the DOI to the publisher's website.

- The final author version and the galley proof are versions of the publication after peer review.

- The final published version features the final layout of the paper including the volume, issue and page numbers.

Link to publication

\footnotetext{
General rights rights.

- You may freely distribute the URL identifying the publication in the public portal. please follow below link for the End User Agreement:

www.umlib.nl/taverne-license

Take down policy

If you believe that this document breaches copyright please contact us at:

repository@maastrichtuniversity.nl

providing details and we will investigate your claim.
}

Copyright and moral rights for the publications made accessible in the public portal are retained by the authors and/or other copyright owners and it is a condition of accessing publications that users recognise and abide by the legal requirements associated with these

- Users may download and print one copy of any publication from the public portal for the purpose of private study or research.

- You may not further distribute the material or use it for any profit-making activity or commercial gain

If the publication is distributed under the terms of Article 25fa of the Dutch Copyright Act, indicated by the "Taverne" license above, 


\title{
Human cortical connectome reconstruction from diffusion weighted MRI: The effect of tractography algorithm
}

\author{
Matteo Bastiani ${ }^{\mathrm{a}, \mathrm{b}, *}$, Nadim Jon Shah ${ }^{\mathrm{a}, \mathrm{b}, \mathrm{c}}$, Rainer Goebel ${ }^{\mathrm{a}}$, Alard Roebroeck ${ }^{\mathrm{a}}$ \\ a Department of Cognitive Neuroscience, Faculty of Psychology and Neuroscience, Maastricht University, Maastricht, The Netherlands \\ ${ }^{b}$ Institute of Neuroscience and Medicine 4, Forschungszentrum Juelich GmbH, Juelich, Germany \\ c Department of Neurology, Faculty of Medicine, RWTH Aachen University, JARA, Aachen, Germany
}

\section{A R T I C L E I N F O}

\section{Article history:}

Accepted 3 June 2012

Available online 12 June 2012

\section{Keywords}

Diffusion weighted magnetic resonance

imaging

Tractography

Human connectome

Structural network analysis

Quality control

Tract specific density coefficient

\begin{abstract}
A B S T R A C T
Reconstructing the macroscopic human cortical connectome by Diffusion Weighted Imaging (DWI) is a challenging research topic that has recently gained a lot of attention. In the present work, we investigate the effects of intra-voxel fiber direction modeling and tractography algorithm on derived structural network indices (e.g. density, small-worldness and global efficiency). The investigation is centered on three semiindependent distinctions within the large set of available diffusion models and tractography methods: i) single fiber direction versus multiple directions in the intra-voxel diffusion model, ii) deterministic versus probabilistic tractography and iii) local versus global measure-of-fit of the reconstructed fiber trajectories. The effect of algorithm and parameter choice has two components. First, there is the large effect of tractography algorithm and parameters on global network density, which is known to strongly affect graph indices. Second, and more importantly, there are remaining effects on graph indices which range in the tens of percent even when global density is controlled for. This is crucial for the sensitivity of any human structural network study and for the validity of study comparisons. We then investigate the effect of the choice of tractography algorithm on sensitivity and specificity of the resulting connections with a connectome dissection quality control (QC) approach. In this approach, evaluation of Tract Specific Density Coefficients (TSDCs) measures sensitivity while careful inspection of tractography path results assesses specificity. We use this to discuss interactions in the combined effects of these methods and implications for future studies.
\end{abstract}

(c) 2012 Elsevier Inc. All rights reserved.

\section{Introduction}

Reconstructing in vivo the macro-scale human connectome (Sporns et al., 2005), which is the map of all the structural connections in the human brain, represents a challenging and important research topic in neuroimaging which has gained a lot of popularity in recent years. An individual large-scale connectome is represented by a graph with a set of nodes, representing a parcellation of the brain anatomy, and a set of edges (equivalently: an edge-weight connectivity matrix or binary adjacency matrix) which can be usefully analyzed using graph theory and network analysis (Rubinov and Sporns, 2010). The main technique by which individual structural connectome reconstruction is made possible in vivo is diffusion-weighted magnetic resonance imaging (DWI).

There are several steps in the post-processing pipeline for DWI data to create a connectivity matrix that represents the macroscopic structural connectome (Hagmann et al., 2007, 2008). Among them are white matter

\footnotetext{
* Corresponding author at: Department of Cognitive Neuroscience, Maastricht University, Uns40 East r 5.755, The Netherlands.

E-mail address: matteo.bastiani@maastrichtuniversity.nl (M. Bastiani).
}

and cortical gray matter segmentation from an anatomical (T1-weighted) MRI, parcellation of the gray matter into nodes of the connectome graph, creation of white matter seed points and masks, intra-voxel water diffusion and/or fiber direction modeling, tractography to connect local directions into long-distance connections and computation of edgeweights between cortical nodes from tractography results. Earlier work has investigated the effects of gray matter parcellation (Zalesky et al., 2010), seed point placement strategy for tractography (Cheng et al., 2011; Li et al., 2011) and the reproducibility of network indices (Bassett et al., 2011a). For instance, Zalesky et al. (2010) show that the small-worldness measure increases with a larger number of nodes resulting from a greater degree of parcellation of the gray matter. In $\mathrm{Li}$ et al. (2011), it is shown that the identification of network hubs in the brain can depend on the tractography seed point placement strategy.

Here we focus on the effects of intra-voxel fiber direction modeling and tractography algorithm on connectome graphs, each of which has seen intensive development and a plethora of approaches over the last decade. We center this investigation on three semi-independent distinctions within this large set of available methods, as summarized in Table 1: i) a single fiber direction compartment versus multiple directions in the intra-voxel diffusion model, ii) a deterministic versus a probabilistic tractography algorithm and iii) a local or step-wise 
Table 1

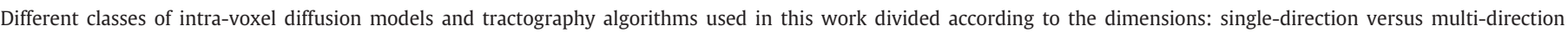

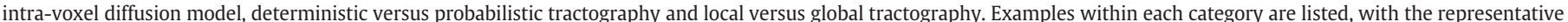

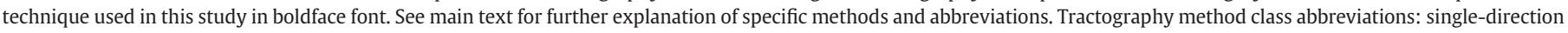

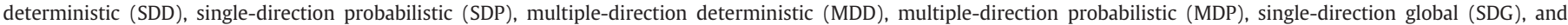

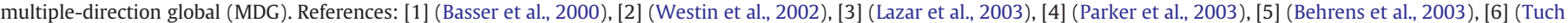

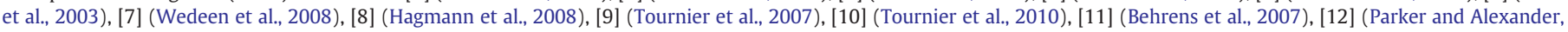
2005), [13] (Iturria-Medina et al., 2007), [14] (Sherbondy et al., 2008), [15] (Sotiropoulos et al., 2010), and [16] (Sherbondy et al., 2009).

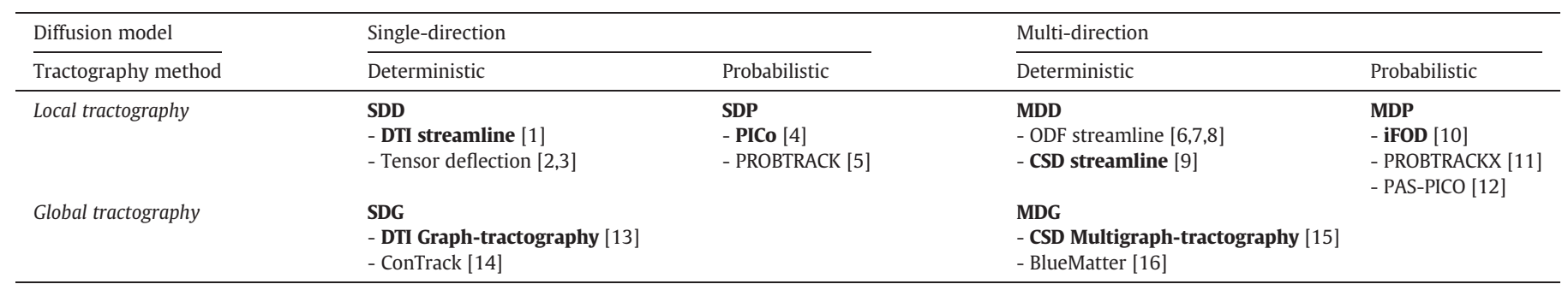

versus global measure-of-fit to the voxel-wise data in reconstructing fiber trajectories.

Several models have been proposed to characterize intra-voxel water diffusion hindered or restricted by white matter fiber tissue, based on the diffusion-weighted MR signal. Diffusion tensor imaging (DTI; Basser and Pierpaoli, 1996; Basser et al., 1994) represents the most established post-processing technique for DWI data. It consists of fitting a rank-2 tensor in each imaging voxel to model the molecules' diffusion as a three dimensional Gaussian probability distribution function (PDF). The uni-modal Gaussian PDF admits only a single main direction of diffusion in a voxel.

It was recognized early on that being able to estimate a single fiber direction per imaging voxel might not be enough. This is because partial volume effects will average two or more fiber populations with different local orientations in large parts of the imaging volume. Furthermore, fibers might be tightly interdigitated, producing something more like a 3-dimensional weave than multiple tight fasciculations. This has stimulated investigation into new acquisition protocols, such as high angular resolution diffusion imaging (HARDI; Frank, 2001; Tuch et al., 2002), and new PDF reconstruction strategies that can produce multi-modal functions. Initially diffusion spectrum imaging (DSI; Wedeen et al., 2005) and Q-ball imaging (QBI; Tuch, 2004; Tuch et al., 2003) were posited as techniques that estimate the actual spin displacement of water molecules without making any prior assumption about the shape of the 3D PDF. A crucial abstraction made in QBI was to model only the 2D orientation distribution function (ODF) on the sphere. The identification of more than one diffusion direction in a voxel enables superior fiber tractography through regions with multiple populations of fibers with different orientations (Wedeen et al., 2008). Subsequently, many more methods have been proposed to identify multiple fiber directions per voxel, including constrained spherical deconvolution (CSD; Tournier et al., 2004, 2007), the extended ball and stick model (Behrens et al., 2007), the diffusion orientation transform (DOT; Ozarslan et al., 2006), the composite hindered and restricted model of diffusion (CHARMED; Assaf and Basser, 2005), generalized diffusion tensors (Liu et al., 2004; Ozarslan and Mareci, 2003) and generalized q-sample imaging (GQI; Yeh et al., 2010).

Once the fiber directions have been estimated for each voxel, tractography algorithms aim to reconstruct the axonal fiber bundles connecting different brain areas. The first tractography approaches to be developed connect discrete steps along the preferential diffusion directions (Basser et al., 2000; Conturo et al., 1999; Mori et al., 1999), the so-called deterministic 'streamline' approach. So-called tensor deflection or tensor projection was introduced to use more than just the primary eigenvector of the local diffusion tensor model (Lazar et al., 2003; Westin et al., 2002). The streamline approach was soon generalized to multi-direction fiber models (Tuch et al., 2003).
Probabilistic tractography approaches characterize the variability of tractography results arising from the uncertainty of the estimated local fiber directions (Behrens et al., 2003; Parker et al., 2003). This contrasts them with deterministic approaches which give a single deterministic answer for the connection of a given region-of-interest (ROI) to any part of the brain. More precisely, the result of a probabilistic tractography algorithm for every single well-defined seed point is a 3D map of visitation counts for fibers through a voxel. The same noisy streamline or diffusing particle principles have been applied to multidirection models to create what we call local probabilistic multidirection approaches (Behrens et al., 2007; Parker and Alexander, 2005; Tournier et al., 2010).

A final recent development step is to move from local step-wise reconstruction of fiber trajectories to a global goodness-of-fit of the entire candidate fiber. Here, the measure of fit quantifies the joint likelihood of the fiber given all voxel data it passes through (Jbabdi et al., 2007; Sherbondy et al., 2008, 2009; Tuch et al., 2002; Zalesky and Fornito, 2009). The global fit measure makes tractography less sensitive to modeling errors caused by local noise (Jbabdi et al., 2007). Recently, a graph-based tractography algorithm (Iturria-Medina et al., 2007) and its extension to a multiple direction fiber models (Sotiropoulos et al., 2010) have been proposed. These algorithms reconceptualize the global tractography problem as a shortest-path search in a graph, in which (in contrast with the connectome graph, described above) nodes are represented by the center of each white-matter voxel. Since graph-weights are then defined as the probability of voxel-center connections given the local ODFs, a shortest path from one point to another in this graph constitutes a globally optimized fiber. Since in a shortest-path search all possible nodes are visited and the path lengths recorded, the n\% shortest paths then correspond to the n\% most likely paths in the probabilistic tractography sense. Thus, graph-based methods - and in fact, global methods in general - are naturally used as probabilistic methods. The only way to force a global graph based approach to be deterministic is to select only its highest percentile results for consideration, which corresponds to looking at only the single shortest path that connects two points. This is why in Table 1 , global methods are considered to be probabilistic, with the possibility of a deterministic interpretation when only the highest percentile results are considered.

The first aim of this paper is assessing the effects of two different intra-voxel diffusion model classes and four different tractography algorithm classes (see Table 1) on the whole-brain connectivity matrix. To this end we evaluate the outcome of applying all method combinations to construct a cortical connectivity matrix. Commonly used graph measures are then calculated to evaluate their sensitivity to the employed diffusion model and tractography algorithm. This means that the question on which tractography approach to choose for in vivo connectome reconstruction becomes even more important. 
Therefore, in the second part of this paper we present a connectome dissection quality control (QC) approach that combines careful inspection of tractography path results with tract specific density coefficients (TSDCs) that quantify sensitivity and specificity.

\section{Methods}

\section{MR data acquisition}

Whole brain scans of two healthy male subjects (aged 25 and 29) were acquired after obtaining written informed consent. Previous investigations have shown that the reproducibility of structural network indices over different subjects is very high (Bassett et al., 2011a). In addition, we investigate intra-subject reliability by computing 10 different random surface parcellations (Zalesky et al., 2010) for each subject (described below). The experiment was approved by the local ethical committee of the Faculty of Psychology and Neuroscience.

A Siemens 3 T MAGNETOM Allegra MR scanner equipped with a high slew-rate head gradient-coil (amplitude $40 \mathrm{mT} / \mathrm{m}$, slew rate $400 \mathrm{~T} / \mathrm{m} / \mathrm{s}$ ) and an 8-channel phased-array head RF-coil was used to acquire the data. A double refocused spin-echo diffusion sequence was used to acquire 131 volumes of data, with $\mathrm{TR}=6600 \mathrm{~ms}$, TE $=$ $94 \mathrm{~ms}$, b-value $=3000 \mathrm{~s} / \mathrm{mm}^{2}, 88 \times 88$ matrix, 52 axial slices, $2.5 \times$ $2.5 \times 2.5 \mathrm{~mm}^{3}$ voxels, partial Fourier $=6 / 8$ and a bandwidth of $2840 \mathrm{~Hz} /$ pixel (echo-spacing $0.4 \mathrm{~ms}$ ). A total of 120 diffusion gradient directions were acquired with 11 unweighted $\left(b=0 \mathrm{~s} / \mathrm{mm}^{2}\right)$ volumes acquired after every 12 gradient directions and including the first and last volumes. The total acquisition time for the diffusion weighted data was $14: 30 \mathrm{~min}$.

A T1-weighted 3D MPRAGE scan ( $\mathrm{TR}=2250, \mathrm{TE}=2.6 \mathrm{~ms}$, flip angle $=9^{\circ}, 256 \times 256$ matrix, 192 sagittal slices, $1 \times 1 \times 1 \mathrm{~mm}$ voxels) was acquired for gray/white matter boundary segmentation. Resting state fMRI data was also acquired in the same session (not reported here). The subject's head was immobilized using foam cushions.

\section{Diffusion data preprocessing}

Diffusion data were preprocessed in order to correct for bulk motion and distortions induced by eddy currents using the FMRIB's Diffusion Toolbox available in FSL (Behrens et al., 2003). The estimated transformation matrices were used to transform the diffusion gradient directions accordingly (Leemans and Jones, 2009). No further transformations were performed on the diffusion-weighted data (or on the diffusion models estimated from it, see below). That is: all directional modeling and tractography were performed in 'native' diffusion data space.

All algorithms use the same white matter masks or waypoint maps, through which the fibers were allowed to pass. In order to obtain these maps, fractional anisotropy (FA) volumes were thresholded using two values and a $3 \times 3 \times 3$ median filter was applied to remove any holes. To investigate the effect of FA threshold, two versions were created at values of 0.1 and 0.2 .

In the current study, we did not correct for EPI distortions by a field map correction. In this head scanner with high slew rate capability, the geometric distortion for diffusion imaging from EPI was found to be relatively small compared to $2.5 \mathrm{~mm}$ isotropic voxels. Field map correction was evaluated but was found not to lead to a significant improvement in distortions.

\section{Anatomical data processing}

White matter (WM), gray matter (GM) and cerebrospinal fluid (CSF) were segmented using FSL FAST (Zhang et al., 2001). The white matter volume was then imported in BrainVoyager QX (v. 2.2; Goebel et al., 2006) for further refinement and creation of a 100,000 vertex tessellation of the WM/GM boundary of both hemispheres using the surface reconstruction algorithm. The boundary surface was transformed to the DWI data space by an affine transform to determine the termination point of white matter fibers at the gray matter boundary. By moving inward from the WM/GM boundary along the surface normal to the closest WM mask voxel, 30.000 unique WM/GM boundary (WMbound) voxels were identified in diffusion data space. These Wmbound voxels were considered as termination loci when reconstructing the high-resolution connectome.

A lower-resolution connectome was obtained from the highresolution one by parcellating the WM/GM surface in 4000 random equi-surface patches (the connectome graph nodes) using a modified iterative constrained centroidal Voronoi tessellation (CCVT) approach (Du et al., 2003) which is described in more detail in Appendix A. The number of 4000 was motivated by Zalesky et al. (2010) and the aim to use a large number of equi-surface parcels that simultaneously minimizes the effect of parcellation and maximizes the sensitivity to the effects of tractography approach. To further decrease the effect of any single random parcellation all results presented are averaged over 10 different random parcellations per subject. Standard errors are computed over all 10 parcellations and over both subjects.

\section{Voxel-wise diffusion model estimation}

In this paper, two models representing two general classes of intravoxel diffusion and fiber architecture were used. As a single-direction model, diffusion tensors (DTs) were fitted to the acquired data by linear regression using a least-square minimization approach. The tensorial matrix was then diagonalized by a Jacobi rotation technique to obtain its orthogonal eigenvector system.

As a multi-direction HARDI based model, CSD fiber orientation distributions (FODs) were reconstructed (Tournier et al., 2007) over a five-fold tessellated icosahedron. This technique was selected for its robustness in estimating orientational distributions from HARDI data. Moreover, FODs represent actual fiber orientation distributions rather than water-bound spin displacements, which leads to stable and accurate local orientations that are very beneficial for both local and global tractography purposes (Fillard et al., 2011). Subsequently, up to three rank-1 6th-order tensors were fitted to every FOD (Schultz and Seidel, 2008) in order to extract up to three main fiber directions in each voxel. This technique allows estimation of the actual fiber orientations without directional bias from the spherical tessellation used when estimating the FODs.

All algorithms have been implemented in custom-written $\mathrm{C}++$ code and in MATLAB (R2009b, The MathWorks, Natick, Massachusetts, USA).

\section{Tractography algorithms and parameters}

To evaluate the different algorithm performances and the changes in the reconstructed connectivity matrices, two parameters were varied: FA and maximum angle (minimum radius of curvature) between two consecutive steps. The step size was set to $1 \mathrm{~mm}$ for all local tractography methods, while for the graph-based global algorithms the step size varies according to the neighborhood size used which, in this work, has been set to $5 \times 5 \times 5$ voxels (see below).

Two angular thresholds were used in this work: $30^{\circ}$ and $90^{\circ}$ which cover the extremes of a realistic range. Thirty degrees represents a very common choice in the diffusion imaging literature, especially when using deterministic algorithms. The value of $90^{\circ}$ widens the possibilities, allowing fibers to turn at very sharp angles. Given the anatomical variability, signal-to-noise ratio (SNR) and the size of the imaging voxels with respect to white matter microstructure, it is difficult to justify any specific angle threshold on a neuro-anatomical basis. Therefore, since this parameter is almost ubiquitously used in tractography, it is important to explore the effect of two extreme values. 
FA maps were thresholded at two different values which are commonly used in the literature $(0.1$ and 0.2$)$ in order to obtain two different WM waypoint masks. These are binary masks containing only those voxels where fibers are allowed to propagate. Since all the tractography algorithms investigated in the present study were run in original diffusion data space, we have chosen to use median filtered FA masks computed in that same space, instead of white matter masks obtained from T1-weighted volumes segmentation, in order to achieve maximum integrity and alignment of WM masks to the diffusion data. To avoid influences on FA such as partial volume effects at the WM/GM boundary and in those voxels where more than two diffusion directions are reconstructed a 3-dimensional median filter has been applied to the thresholded WM volumes to fill holes in the masks. Furthermore, in the WM binary masks, WMbound voxels were always included in the volume after having thresholded the FA mask and used the median filter. Since surface parcellation is performed in anatomical space, alignment is still needed to define WMbound voxels in diffusion space. However, this procedure is slightly more tolerant of misalignment and distortions due to susceptibility gradients since we coregister the surface points from anatomical space to diffusion space by inward projection along the surface normals.

Local deterministic algorithms were started both from a Cartesian $3 \times 3 \times 3$ grid of seed points ( $0.83 \mathrm{~mm}$ spacing) in the interior volume of every WM mask voxel and from those voxels which only belong to the WMbound. All resulting tractography streamlines were then investigated for intersection, on two opposite sides of the seed point, with the WM boundary volume (i.e. all WMbound voxels). All probabilistic methods and global methods were started from all WM boundary voxels, which is necessary for the resulting visitation maps to be interpreted as connecting seed points at the WM/GM boundary to other WM/GM boundary points. In addition, when individual probabilistic streamlines are investigated rather than visitation maps, Li et al. (2011) show that distance related bias can increase when seeding from the entire deep white matter.

For all local methods, deterministic or probabilistic, fibers shorter than $10 \mathrm{~mm}$ or longer than $200 \mathrm{~mm}$ were removed. Furthermore, looping fibers (i.e. fibers that return to already explored voxels) are excluded from the analysis. For global algorithms by the very nature of the shortest path finding approach, loops are automatically excluded.

\section{Local single-direction deterministic (SDD)}

Deterministic streamlines were obtained using Euler stepping integration of the local direction field. Local direction finding takes into account the first eigenvector based on the estimated rank-2 tensor. Nearest neighbor interpolation of the entire diffusion tensor was performed. At every starting seed point, a streamline was initiated parallel to the local primary eigenvector. Deterministic local single-direction tractography was performed with custom-written $\mathrm{C}++$ code (c.f. Roebroeck et al., 2008).

\section{Local single-direction probabilistic (SDP)}

The local streamline-based approach to compute probabilistic indices of connectivity (PICo) as proposed by Parker et al. (2003) was used. This approach estimates the uncertainty in the diffusion direction estimation obtained by DTI and computes the connectivity strength between two regions using Monte Carlo methods. The number of iterations, which is the number of streamlines initiated from each seed point was set to 3000 . The look-up table for orientation sampling matched to FA was computed using the Bingham distribution. Probabilistic local single-direction tractography was performed with the Camino software package (Cook et al., 2006).

\section{Single-direction global (SDG)}

Graph-based tractography as described by Iturria-Medina et al. (2007) was performed. The 3D neighborhood size was set to $5 \times$ $5 \times 5$ for each voxel to be connected to 98 nearest neighbors as edges in the tractography graph. This samples the fitted DT ellipsoid along 98 unique directions using the entire solid angle of $2 * \mathrm{pi} / 98$ to compute edge weights. Connection's strength between two WMbound voxels is computed by multiplying the connection weights along the shortest path which connects them. Global single-direction graph tractography was performed with custom-written $\mathrm{C}++$ code.

\section{Local multi-direction deterministic (MDD)}

Deterministic multi-direction streamlines were obtained using a local co-linearity based approach (Hagmann et al., 2008; Wedeen et al., 2008). That is, local direction finding consisted in following the local direction most colinear to the current fiber tangent. Nearest neighbor interpolation of the multi-direction field was performed. At every starting seed point, streamlines were initiated parallel to every local direction independently (i.e. maximally $27 \times 3$ streamlines per voxel). Deterministic local multi-direction tractography was performed with custom-written $\mathrm{C}++$ code.

\section{Local multi-direction probabilistic (MDP)}

The employed algorithm uses orientations sampled from the FOD at each step and initializes a great number of streamlines per seed point in a way similar to the PICo algorithm. Per seed point, 3000 streamlines were initiated within a sphere whose center corresponds to the center of every WM boundary voxel and whose radius has been set to half the voxel size $(1.25 \mathrm{~mm})$. Probabilistic local multi-direction tractography was performed using the MRtrix package (Tournier et al., 2007).

\section{Multi-direction global (MDG)}

When dealing with multiple directions extracted in every voxel, the concept of a tractography graph (Iturria-Medina et al., 2007) can be extended to the one of a multi-graph (Sotiropoulos et al., 2010). In this case, the center of every voxel is not just a single node, but a collection of $j$ nodes, where $j$ is the number of directions extracted in a specific voxel. Therefore, the weight of an edge connecting two neighboring voxels will not be a scalar anymore, but a $j \times m$ matrix, where $j$ and $m$ are the number of directions extracted in the two considered voxels. Note that although the graph-based algorithms used here (Iturria-Medina et al., 2007; Sotiropoulos et al., 2010) do not account for edge length in the local edge weight calculation, this could be included in the algorithm (Zalesky, 2008). As for single-direction global tractography, the 3D neighborhood size was set to $5 \times 5 \times 5$ for each of the $j$ direction voxel to be connected to $98 * j * m$ nearest fiber-component neighbors. This samples the fitted higher-order tensor along 98 unique directions using the entire solid angle of $2 *$ pi/98 to compute edge weights. Multi-graph tractography was implemented in custom-written $\mathrm{C}++$ code.

\section{Connectivity matrix weight computation and graph analysis}

To move from a very high resolution tractography result that connects all $\sim 30 \mathrm{k}$ voxels in the WMbound voxel set to the $4000 \times 4000$ binarized adjacency matrix, the following steps were followed. For the deterministic algorithms, any streamline connecting any voxel in one parcel to any voxel in another, connects the two parcels in the final adjacency matrix (c.f. Zalesky et al., 2010). For the probabilistic and global algorithms the threshold on percentile visitation counts or percentile graph weights was varied (between $80 \%$ and 99\%) to investigate the effect of probabilistic thresholding. Furthermore, the result from a probabilistic method is asymmetric (the count or weight for A seeded from B is not necessarily equal to the count or weight for B seeded from A). Thus, any non-zero count or weight connecting any voxel in one parcel to any voxel in another in either of the two directions, connects the two parcels in the final symmetric adjacency matrix (c.f. Iturria-Medina et al., 2011). These binarization steps to construct the final $4000 \times 4000$ adjacency matrix are aimed at 
eliminating the effect of an arbitrary threshold, patch-area normalization or fiber-length normalization. For each binarization the edge density is computed as $K /\left(\left(N^{2}-N\right) / 2\right)$ with $K$ the number of supra-threshold edges and $N$ the number of nodes, which here is always 4000 .

Computation of graph measures was performed using the Brain Connectivity Toolbox (Rubinov and Sporns, 2010). The small-worldness index is defined as the ratio of the normalized averaged clustering coefficient with the normalized path length of the considered graph (Watts and Strogatz, 1998). To normalize the average clustering coefficient and the path length of a network means to compare both measures with those obtained from a random network which is matched in terms of degree distribution. Given the size and the numbers of the graphs used in the present study, the approach proposed by Zalesky et al. (2010) was used, in which the average clustering coefficient and the path length of a random network was estimated using the analytical formula for an equivalent Erdös-Rényi random graph. A connectome is said to be small-world if the ratio of the two normalized indexes is higher than one, with the normalized clustering coefficient larger than one and the normalized path length close to one.

\section{Connectome dissection quality control}

We suggest an empirical approach to quality control the tractography results that uses connectome dissection. The connectome is partly dissected into a few large associative bundles which are known to exist. These are selected by specifying two cortical end ROIs (c.f. Catani and Thiebaut de Schotten, 2008; Ffytche et al., 2010). The choice of tracts and end ROIs partly dissects the connectome adjacency matrix into a few submatrices, each of which corresponds to the selected bundle (Cammoun et al., 2012). If anatomical expertise is used to select the end ROIs carefully, one can now use the density (patch-to-patch connection count) of each of these submatrices as an empirical index of sensitivity. We define the tract specific density coefficient (TSDC) as:

$\operatorname{TSDC}=\frac{K_{\left\{N_{i}\right\},\left\{N_{j}\right\}}}{\left|N_{i}\right| \cdot\left|N_{j}\right|}$

where $N_{i}$ and $N_{j}$ are the sets of nodes (cortical patches) corresponding to the $i$-th and the $j$-th regions of interest, respectively, $|N|$ is the number of nodes in a set and $K_{\left.\left\{N_{i}\right\},\{N\}\right\}}$ is the count of non-zero entries of the submatrix of the whole brain adjacency matrix defined by the two node sets. The TSDC always ranges from 0 to 1 and quantifies the inferred strength of a known tract as expressed in the whole brain adjacency matrix. 0 signifies absolute absence of inferred connections (low sensitivity in case of a known tract). Since we normalize by $\left|N_{i}\right| \cdot\left|N_{j}\right|$, the maximum value of 1 would mean that every single patch in an ROI is connected to every single patch in the second ROI. This kind of complete mutual convergence/divergence is neurophysiologically not necessarily plausible in large associative cortico-cortical projections. This means that although the target for a known connection is a TSDC considerably higher than 0 , one would normally not want a TSDC that approaches 1 . As a convention, we suggest here that a TSDC as a QC measure for sensitivity (a sensitivity TSDC, to be contrasted with a specificity TSDC, see below) should range between 0.1 and 0.5 for a cortical parcellation with 4000 nodes. Although the normalization does not change the result of any comparison between two different tractography methods for the same tract, it crucially enables absolute interpretation of TSDCs and comparisons between different tracts. Conceivably, it could be used to quality control entire connectome matrices by requiring TSDCs in a certain range for a considerable number of known tracts, which together span a large fraction of the adjacency matrix.

Here there are three important issues. First, as its name implies, a TSDC is very specific to a tract and the accurate expert identification of its end ROIs. Comparing algorithms with each other on the basis of TSDCs will require looking at a couple of tracts. Here we have chosen three $\left(\mathrm{TSDC}_{\mathrm{CC}}, \mathrm{TSDC}_{\mathrm{SLFII}}\right.$ and TSDC $\mathrm{ILF}$ ) that together cover an important subset of the challenges that tractography algorithms face. The ROIs for selecting these three bundles were derived from the literature (Catani and Thiebaut de Schotten, 2008; Ffytche et al., 2010; Wakana et al., 2004) and manually selected on the reconstructed WMbound surface. After a first manual selection, the ROIs are then constrained by the actual parcellation used for the definition of the nodes of the connectome, so that the refined ROI only contains those cortical patches which are entirely comprised in the first selection. For the CC we selected a triangular patch whose vertices are given by the anterior part of the superior frontal gyrus, the dorsal part of the precentral gyrus and the inferior frontal gyrus. For the SLFII we selected patches belonging to the dorsolateral prefrontal cortex and the inferior parietal cortex as outlined in Ffytche et al. (2010) and Thiebaut de Schotten et al. (2011). Note that there may be hemispheric differences (rightward asymmetry) in the SLF II (Ramayya et al., 2010); much like those that have been found repeatedly for the arcuate fasciculus (leftward asymmetry). Here, we have only selected the ROIs lying on the left hemisphere. Finally, for the ILF we selected patches lying on the anteriormost part of the temporal lobe (temporal pole) and on the posteriormost part of the occipital lobe as outlined in Catani and Thiebaut de Schotten (2008) and Wakana et al. (2004). Second, the behavior of TSDC is influenced by the choice of the number of nodes that span the cortex obtained by the cortical parcellation. High values approaching 1 are more easily obtained for smaller number of nodes and coarser cortical parcellation since tract specific submatrices of the connectome adjacency matrix become denser (Cammoun et al., 2012). Third, TSDCs for a set of tracts as an evaluation of sensitivity must be balanced by also evaluating specificity. This must be done in at least two ways. We first verify that the TSDC for two ROIs which are known not to be connected (specificity TSDC) is small. This requires identifying a set of pairs of non-connected ROIs. Here, for illustration, we chose a set of subregions of the ROIs used to track the corpus callosum, which causes them to be distance-matched with the corpus callosum tracts. Crucially, we selected the anteriormost part of the superior frontal gyrus in the left CC ROI and the much more posterior precentral gyrus in the right CC ROI. Although heterotopic connections through the CC are known to exist in the frontal cortex (Jarbo et al., 2012), and the precentral gyrus might be connected to the contralateral posteriormost superior frontal gyrus, we would expect a-priori that there are no connections between these two ROIs. Thus, as a first quantitative measure of specificity we require the TSDC computed for these ROIs to be very small. However, this procedure will potentially miss many false positives because even if tracts connect two truepositive ROIs with a high TSDC, the tract paths themselves might take an erroneous course between them. Therefore, secondly we evaluate sensitivity TSDCs for known connections in conjunction with careful inspection of tractography path results to identify erroneous tracking results.

\section{Results}

Fig. 1 shows the edge density of the final adjacency matrices for all methods. The results are very consistent over subjects and parcellations (the standard error computed over subjects and parcellations was maximally 0.001 for local approaches and 0.003 for global approaches across all parameter combinations). Density always decreases with increasing probabilistic threshold: as more of the probabilistic tractography results fall below threshold, final graph sparseness increases. In a similar way, both a change in FA threshold from 0.2 to 0.1 and a change in angle threshold from $30^{\circ}$ to $90^{\circ}$ tend to roughly double the connection density. Generally, as can be expected, more liberal thresholds (lower probabilistic, FA or minimum curvature thresholds) lead to higher global connection density. Less intuitive, but evident in our analyses, global methods tend to have up to twice the connection density of local methods at the same thresholds. The effect of probabilistic versus deterministic approaches is most clearly seen in Fig. 1 at the $30^{\circ}$ angle threshold. As discussed above, one can take a deterministic interpretation of a global 
FA $0.1,30 \mathrm{deg}$

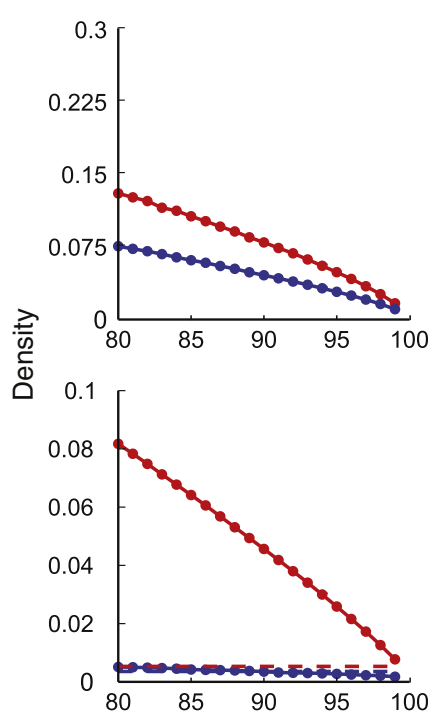

FA $0.1,90 \mathrm{deg}$
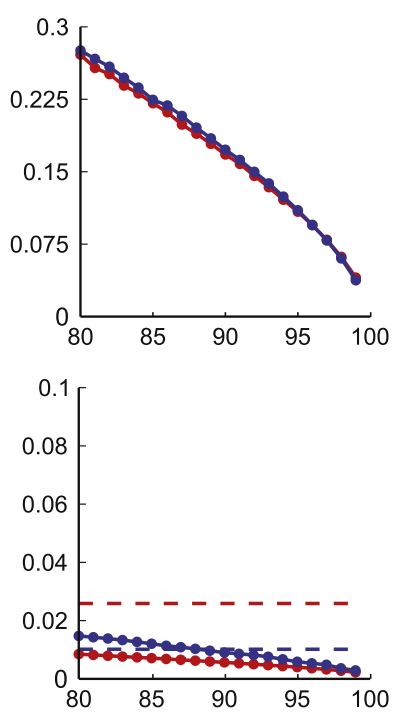

FA $0.2,30 \mathrm{deg}$
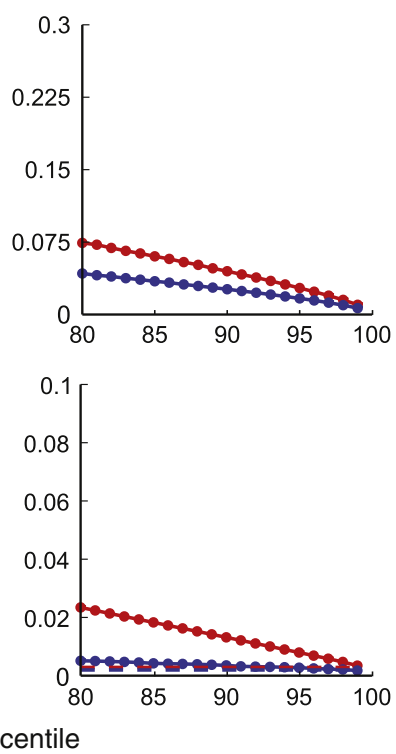

FA $0.2,90 \mathrm{deg}$
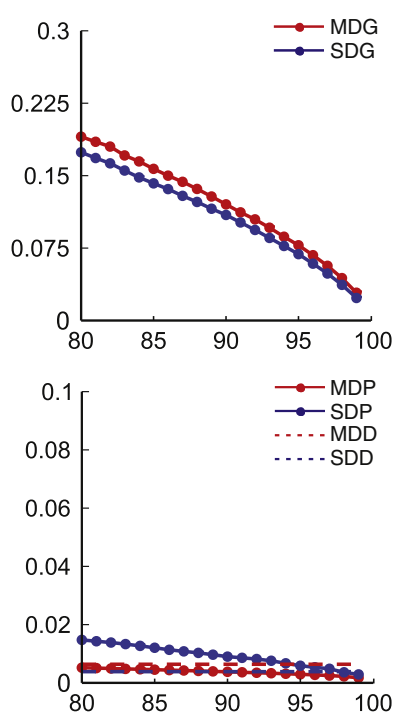

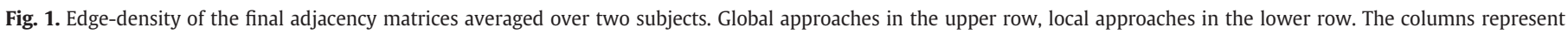

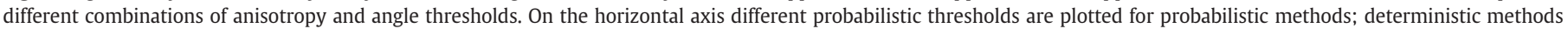
are plotted as a single straight line. Single-direction methods are plotted in blue, multiple-direction methods in red. Note the different scales on the y-axis.

tractography result by considering only the highest percentile results. Interestingly, this 'deterministic limit of probabilistic results' is also found experimentally in the local multi-direction results at a $30^{\circ}$ angle threshold. This is interesting given that (in contradistinction with the global methods) this represents a convergence in results in two separate local tractography approaches.

Local probabilistic methods at very liberal thresholds, especially a $90^{\circ}$ angle threshold in absence of a colinearity constraint based on prior identification of main diffusion directions can lead to degeneration of algorithm performance and pathological results. This manifests itself in the reversal of some of the patterns above, such as a higher density for the single direction method. This degeneracy is especially applicable to local probabilistic multi-direction methods, presumably because of the greater noise sensitivity of these more complex methods in combination with very permissive probabilistic direction sampling.

Since topological graph indices can be strongly dependent on the edge-density of the network (van Wijk et al., 2010), we plot all subsequent results as a function of edge-density. Density generally decreases: i) with increasing probabilistic threshold, ii) when going from probabilistic to deterministic tractography, iii) with increasing FA and decreasing angle thresholds, iv) when moving from global to local tractography, and $\mathrm{v}$ ) when moving from multi direction to single direction models. It is important to only compare indices between density matched graphs in order to assess the true effects of tractography algorithms and parameters on the topology of whole brain adjacency matrices independent of their simple effects on global density as reported above. However, because adjusting the probabilistic threshold generally has an effect both on density and possibly on topology, we have included corresponding figures plotted as a function of percentile threshold in the supplementary material.

Fig. 2 shows deterministic streamline length histograms for single and multi direction methods at different angle thresholds. Note that only streamlines connecting two WMbound voxels are considered and that many more streamlines are started for MDD (in all FOD directions at each seed point) than for SDD (in one direction at each seed point). The first significant aspect is considerable short-length $(<20 \mathrm{~mm})$ streamline abundance, especially for the multi direction method. Although numerous, streamlines of this short length are unlikely to contribute much to the final adjacency matrix, since they will often connect a node-ROI with itself, which is neglected. In other words, one expects longer streamlines to contribute most to adjacency matrix density. Indeed, a larger number of long (between 40 and $160 \mathrm{~mm}$ ) streamlines in these histograms correspond to a higher density in Fig. 1. This is seen prominently in MDD having a greater density than SDD at the same thresholds. The short streamline abundance is considerably tempered by a more liberal angle threshold $\left(90^{\circ}\right)$, leading to less early termination and hence a greater number of longer streamlines.

Fig. 3 shows the small-worldness index of the final adjacency matrices for all methods. First, it is observed that small-worldness (a coefficient larger than one; Watts and Strogatz, 1998) is a very robust phenomenon in human structural networks obtained with DWI tractography. It is observed over all tractography methods and parameter values and its value is very consistent over subjects and parcellations (the standard error computed over subjects and

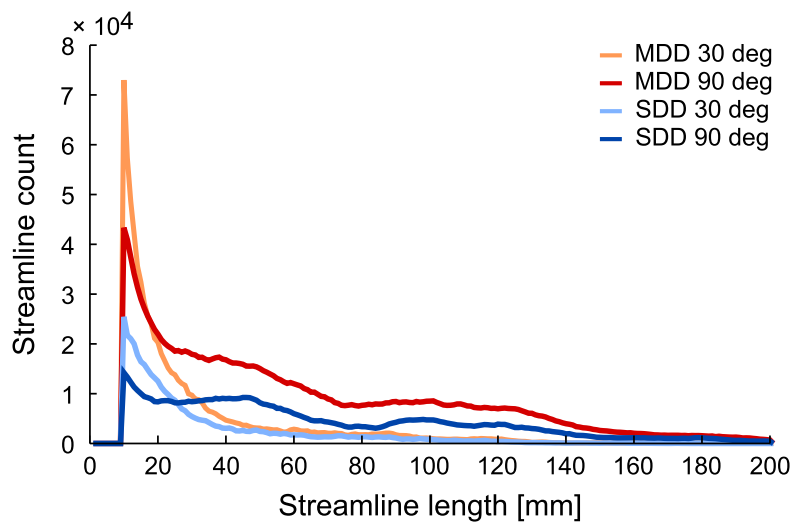

Fig. 2. Length histograms (1 $\mathrm{mm}$ bins) for deterministic streamline tractography methods at FA threshold 0.1 averaged over 2 subjects. Only streamlines connecting WMbound voxels are considered. Note that based streamlines shorter than $10 \mathrm{~mm}$ and longer than $200 \mathrm{~mm}$ are excluded. 
FA $0.1,30$ deg

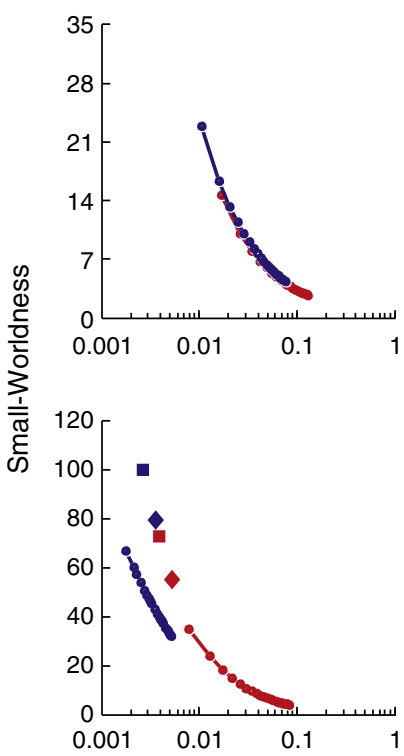

FA $0.1,90 \mathrm{deg}$
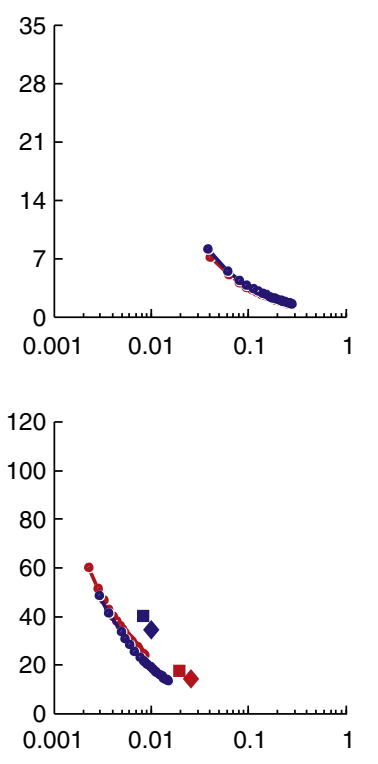

FA 0.2, 30 deg
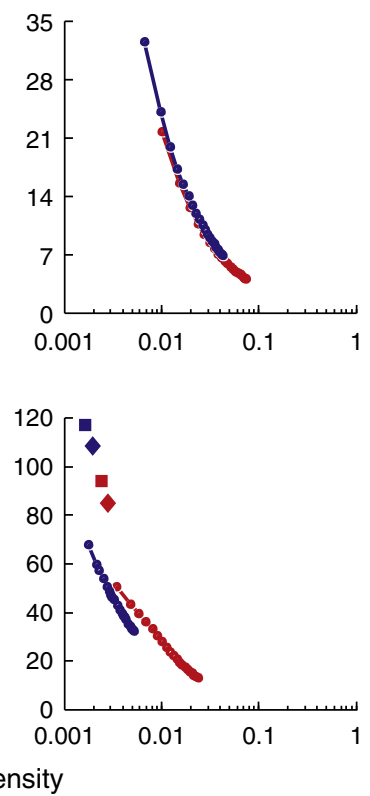

FA $0.2,90 \mathrm{deg}$
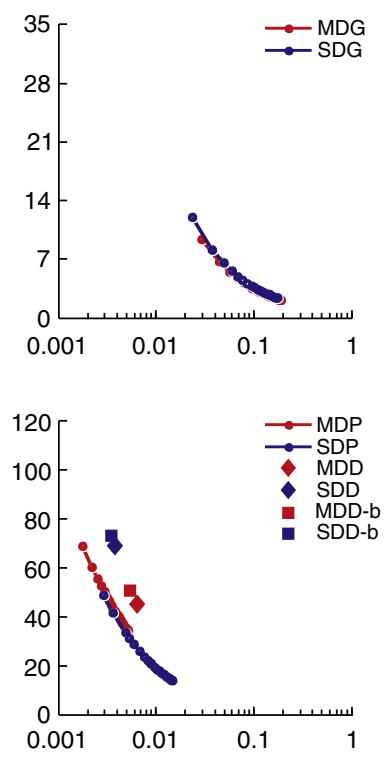

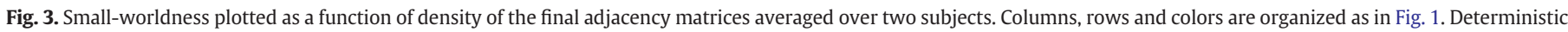

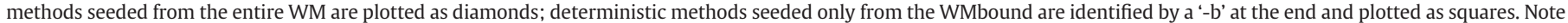
the different scales on the $y$-axis.

parcellations was maximally 3.621 for local approaches and 0.3 for global approaches across all parameter combinations). This agrees with and extends the finding of reproducibility of network indices over scanning session and individuals for local deterministic methods (Bassett et al., 2011b). Considerable effects of tractography algorithm on small-worldness can be observed even when adjacency matrices are density matched. At FA threshold 0.2 and angle threshold $30^{\circ}$, MDD and SDD have coefficients $70 \%$ larger than for density matched SDP. At the same FA and angle thresholds and a density of 0.005 MDP has a coefficient 26\% larger than for density matched SDP. Global versus local differences also are found to be up to $30 \%$, for instance at FA 0.2 and angle 30 and a density of 0.01, MDG and
MDP have small-worldness coefficients of, respectively 21.83 and 28.45. Contrary to these effects of algorithm choice, small-worldness effect to be relatively insensitive to FA and angle thresholds.

The small-worldness coefficient results are best illustrated further by separately inspecting the two components it consists of: normalized path length and normalized average clustering coefficient, reported in Figs. 4 and 5, respectively. Once again, the values for normalized path length and normalized average clustering coefficients are very consistent over subjects and parcellations (with a standard error over subjects and parcellations of maximally 0.093 for local approaches and 0.008 for global approaches for normalized path length, and maximally 1.431 for local approaches and 0.885 for global approaches for normalized
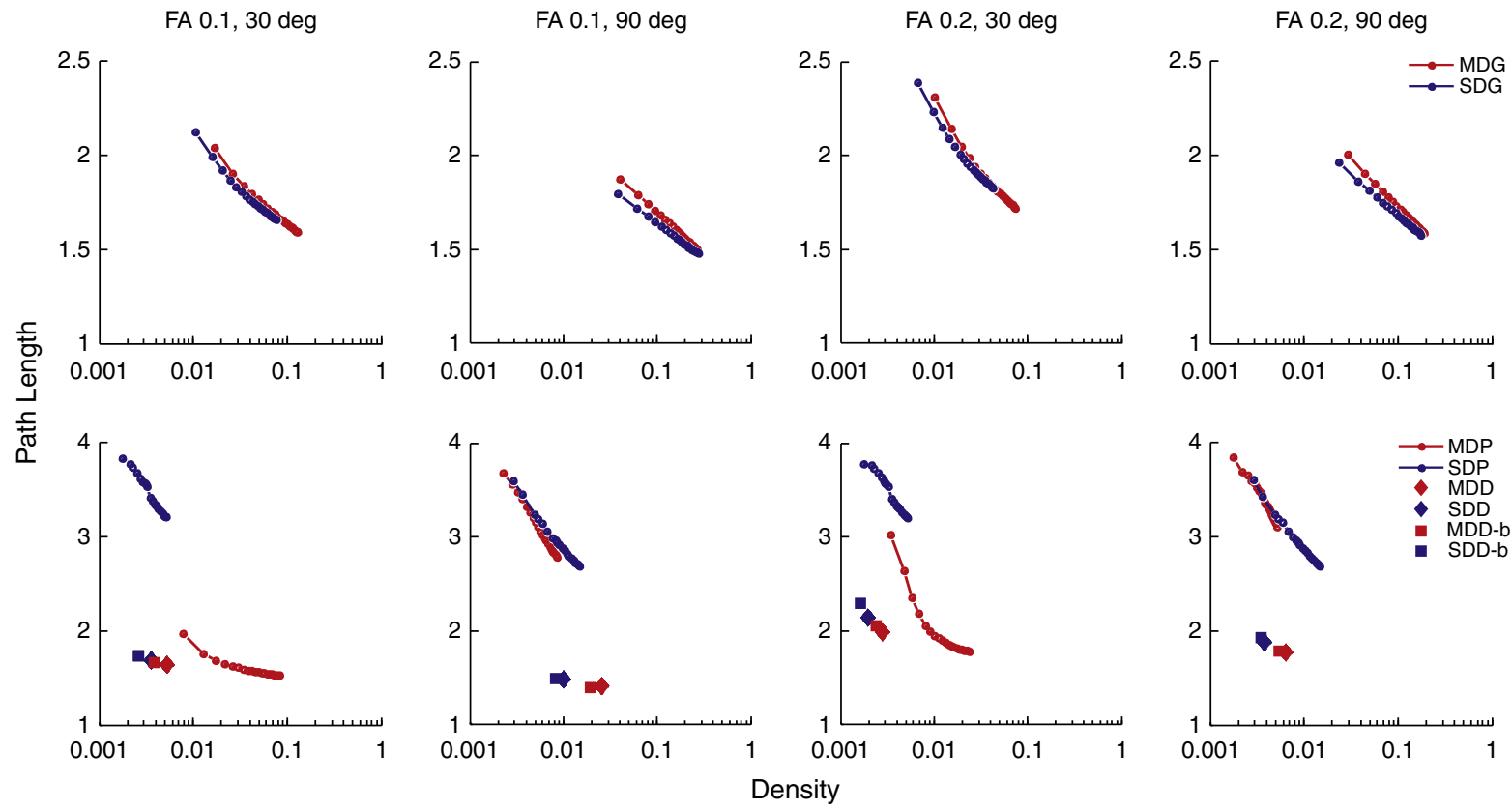

Fig. 4. Characteristic path length averaged over 2 subjects, organized as in Fig. 3. 
FA $0.1,30 \mathrm{deg}$

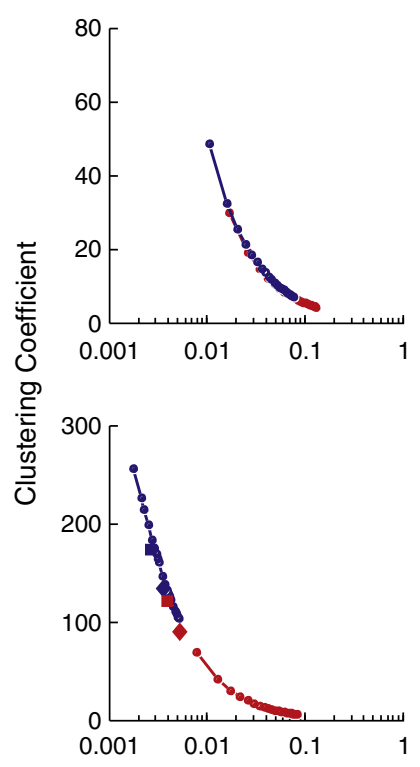

FA $0.1,90$ deg
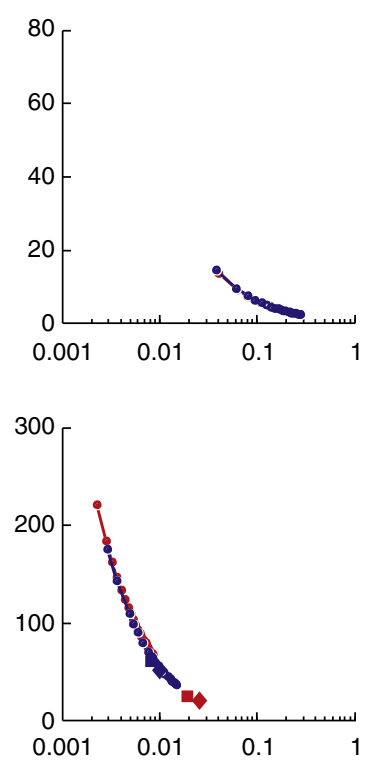

FA $0.2,30$ deg
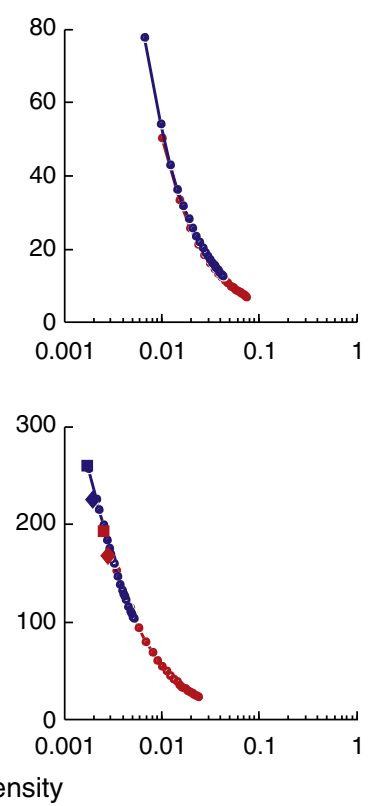

FA $0.2,90$ deg
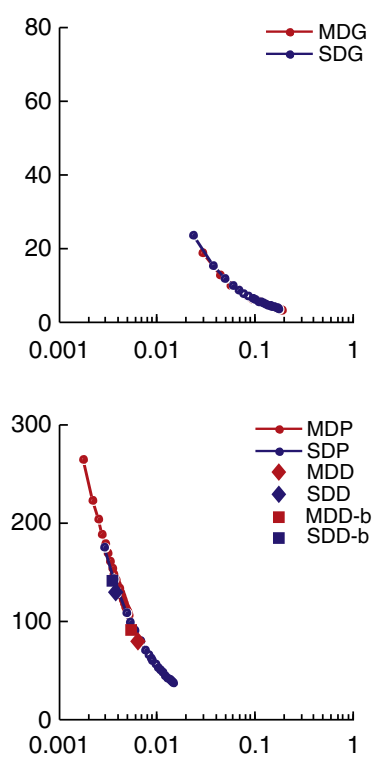

Fig. 5. Average clustering coefficient averaged over two subjects, organized as in Fig. 3.

average clustering coefficients). A larger degree of small-worldness can be caused by a smaller normalized path length, a larger normalized average clustering coefficient or both. For small world networks the normalized path length should be close to one, which is true for most methods and parameter sets, except for the SDP and the MDP for the $90^{\circ}$ angle threshold or for low densities (high probabilistic thresholds). The small-worldness difference of MDD and SDD with density matched SDP is seen to be explained by a normalized path-length for MDD and SDD that is $70-80 \%$ lower than that for SDP. Similarly, there is a $23 \%$ difference between SDP and MDP at FA 0.2 and angle $30^{\circ}$, with normalized path length for SDP larger than for MDP which explains their difference in small-worldness reported above. Finally, the local/global effect in small-worldness can also be traced back to path-length, with 2.3 for MDG and 1.9 for MDP.

Thus, the small-worldness results are largely contained within the effect on normalized path-length, as is indeed supported by Fig. 5 showing a relatively little effect of algorithm choice on clustering coefficient. However, in the low density ranges, where deterministic and probabilistic local approaches can be compared in terms of density and the curve is steeper, differences are still up to $10 \%$. Interestingly, the absence of effect of FA and angle threshold on small-worldness is not explained by the absence of effect on its constituents. Instead, the effect of threshold parameters on both normalized path length and clustering coefficient are large but equal, which leads them to be divided out in the small-worldness coefficient.

Finally, global network efficiency is another graph measure that is often used to quantify the overall shortest path length distribution. It is inversely related to the characteristic path length (Fig. 4). Indeed, Fig. 6 (standard error computed over subjects and parcellations was maximally 0.002 for local approaches and 0.003 for global approaches across all parameter combinations) shows global approaches to be more efficient than local, multi direction to be more efficient than single direction and global network efficiency to increase with increasing density.

For the connectome dissection quality control (QC) we chose to select the second branch of the superior longitudinal fasciculus (SLFII), the inferior longitudinal fasciculus (ILF), both in the left hemisphere, and part of the transcallosal projections (CC) for subject 2 as shown in Fig. 7. Fig. 8 plots $\mathrm{TSDC}_{\mathrm{CC}}, \mathrm{TSDC}_{\mathrm{SLFII}}, \mathrm{TSDC}_{\mathrm{ILF}}$ and $\mathrm{TSDC}_{\mathrm{FP}}$ for four different tractography algorithms and over a range of percentile thresholds for probabilistic algorithms. Here $\mathrm{TSDC}_{\mathrm{FP}}$ is the TSDC for the selected false positive connection between the anteriormost part of the superior frontal gyrus in the left CC ROI and the much more posterior precentral gyrus in the right CC ROI. Note that, in what follows, the probabilistic threshold is always selected on the basis of the whole brain tractography results. Hence, these thresholds are not necessarily equivalent to selecting the nth percentile of paths connecting just the selected ROIs, but they are equal to those applied for the whole brain adjacency matrices. Focusing first on the sensitivity TSDCs, at very low percentile thresholds for CC and SLFI graph-based global algorithms have TSDCs close to 1, whereas $\mathrm{TSDC}_{\mathrm{ILF}}$ for the selected example is an order of magnitude lower for reasons we discuss below. TSDCs for deterministic algorithms range between 0.007 and 0.11 for the selected tracts, with TSDC for MDD being always 2 to 10 times higher than that for SDD for the same tract. Finally, TSDCs for graph based algorithms approach those of deterministic algorithms only for very high thresholds (99th percentile; again we discuss the ILF below). Therefore, we will focus on comparing the tractography results mostly at the intersections of the global and local TSDC curves below. Focusing next on the specificity (false positives) $\mathrm{TSDC}_{\mathrm{FP}}$, pleasingly it shows false positive TSDC scores to be much lower than sensitivity TSDCs. As one would expect for stochastic algorithms when thresholds are low enough, significant false positives start to arise. However it can be seen that for probabilistic thresholds above the 90th percentile false positive TSDC falls below 0.01. Interestingly, this offers a partial solution to choosing a good operating range of probabilistic thresholds, namely that range which simultaneously keeps sensitivity TSDCs between 0.1 and 0.5 and specificity TSDCs below 0.01 . Here this would amount to a threshold between the 91st and the 98th percentiles. Sensitivity TSDCs which fall below 0.1 (as for the ILF here) point to infidelities in tractography results, which illustrate its role as a quality control criterion as discussed below.

Specificity TSDCs will potentially miss false positives because even if tracts connect two true-positive ROIs with a high TSDC, the tract path between themselves might take an erroneous course. Therefore, we continue to evaluate false positives by carefully examining tractography paths for sensitivity TSDCs. We begin our evaluation with the wellknown and often used example of the inter-hemispheric projections through the body of the corpus callosum. It is known that the transcallosal projections connect a large inferior-superior extent of the lateral frontal cortex. This is reflected in our selection of cortical ROIs for the 
FA $0.1,30$ deg

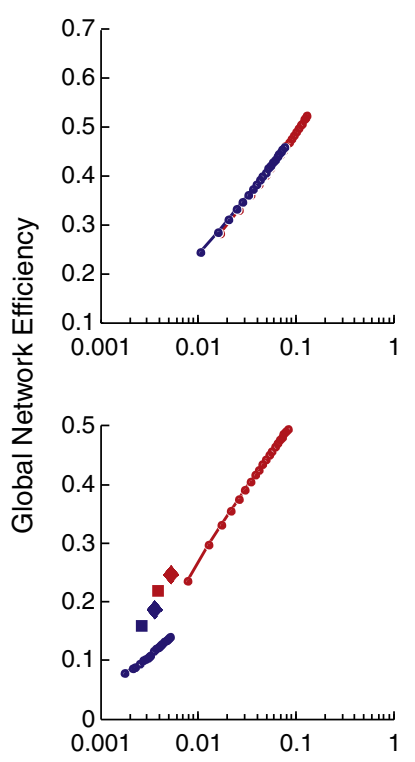

FA $0.1,90$ deg

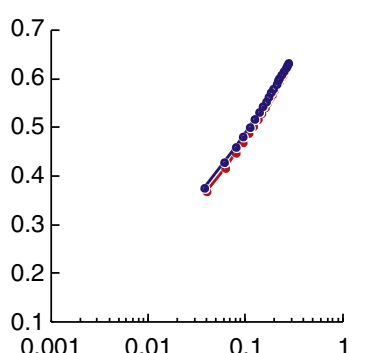

FA 0.2, 30 deg
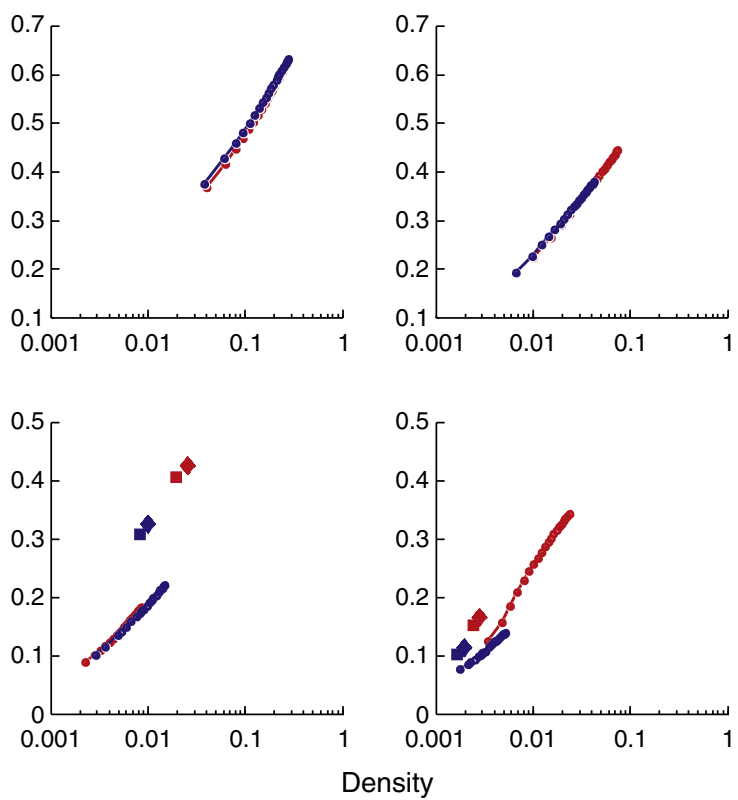

FA $0.2,90$ deg
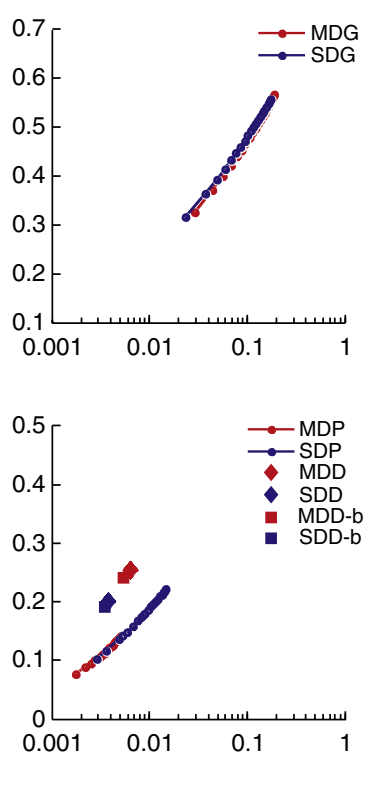

Fig. 6. Global network efficiency averaged over two subjects, organized as in Fig. 3.

CC in Fig. 7 which span the lateral frontal cortical surface (from the lateral superior frontal gyrus to the operculum) for a few centimeters anteriorly to the central sulcus on both hemispheres. These trans-callosal projections pass through the body of the corpus callosum (CC) and cross with several other major fiber tracts. Among these are the superior longitudinal fasciculus (SLF) and the corona radiata (CR), both of which cross and intersect with each other and with transcallosal fibers in the centrum semi-ovale. Thus, the ability of a tractography algorithm to connect the entire inferior-superior extent of the lateral frontal cortex through a three-way crossing is reflected in the TSDC $\mathrm{Cc}_{\mathrm{cc}}$. Fig. 8 shows that the $\mathrm{TSDC}_{\mathrm{CC}}$ is greater for multiple direction approaches than for single direction and greater for global methods than for local methods for all probabilistic thresholds (except the 99th percentile compared to MDD).

To illustrate the difference in the actual streamlines or tractography paths that underlie these differences in TSDC (and global statistics of the adjacency matrices in Figs. 1-6), Figs. 9 and 10 show the corresponding local and global tractography path results. Fig. 9 shows deterministic streamlines for SDD and MDD at a liberal FA threshold and at two angle thresholds (the right-hand 90 degree panels correspond to the $\mathrm{TSDC}_{\mathrm{CC}}$ reported in Fig. 8). The absolute number of streamlines and average lengths for each of the four cases are listed in Table 2 (compare to Fig. 2) and the connected patches on the cortex are reported in Supplementary Fig. S5. Fig. 9 shows two effects which can impact global statistics. First, MDD is more successful in identifying the lateral transcallosal projections (c.f. Tuch et al., 2003; Wedeen et al., 2008), which is confirmed by a higher TSDC $\mathrm{CC}_{\mathrm{C}}$ in Fig. 8. Even at very liberal anisotropy and angle thresholds SDD only identifies a few lateral callosal projections, some of which track wrongly into the SLF or CR along their way. That is, there is a large amount of false negatives in the SDD results, even at liberal thresholds. Second, the MDD results at two different angle thresholds show a classical sensitivity/specificity trade-off. A few of the inferior lateral trancallosal projections which are missed at the $30^{\circ}$ threshold are correctly reconstructed at a $90^{\circ}$ threshold. However, this is at the cost of a large number of false positive streamlines that, again, connect both lateral surfaces to SLF or CR pathways. This sensitivity/specificity trade-off for the transcallosal projection is improved further in the corresponding SDG and MDG results shown in Fig. 10 with the corresponding connected cortical patches reported in Supplementary Fig. S6. Here, results are shown for the same liberal thresholds on FA and angle as Fig. 8 and in the right side of Fig. 9, now at three different probabilistic thresholds. Note that, because of virtually equal density

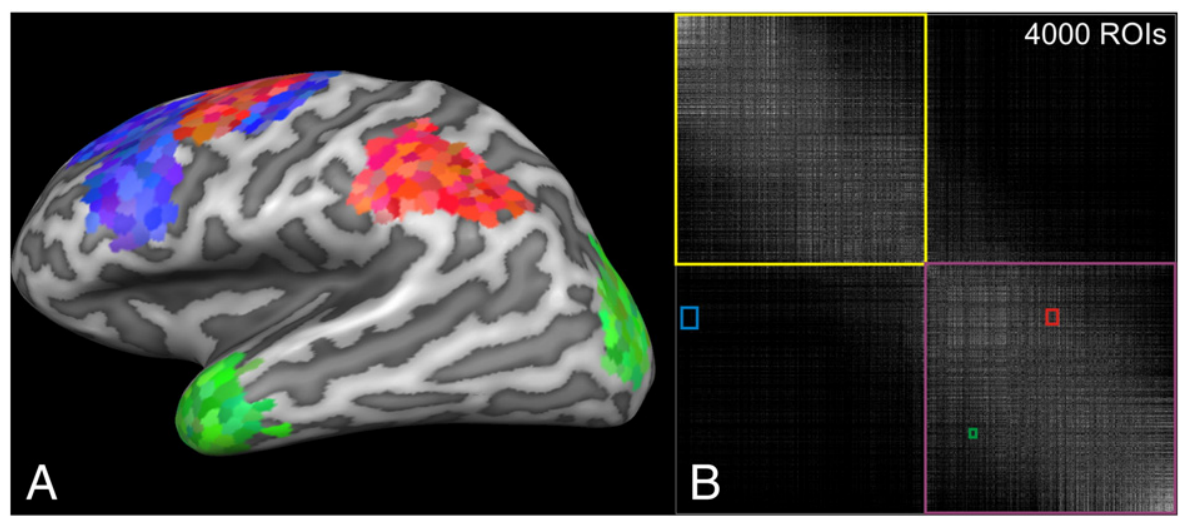

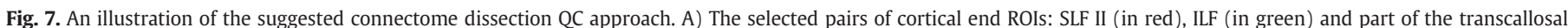

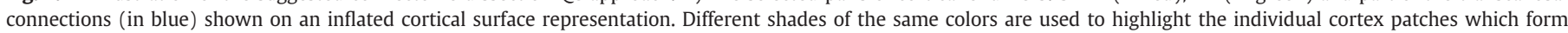

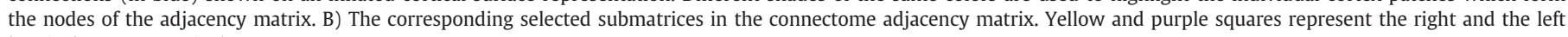
hemispheres, respectively. 
$\mathrm{CC}$

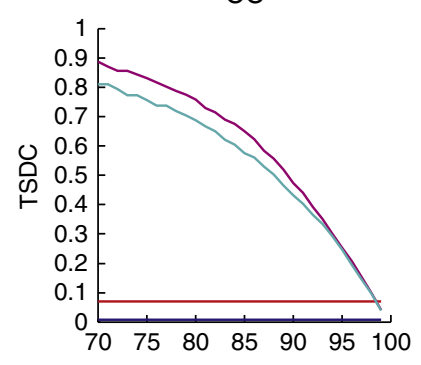

SLFII

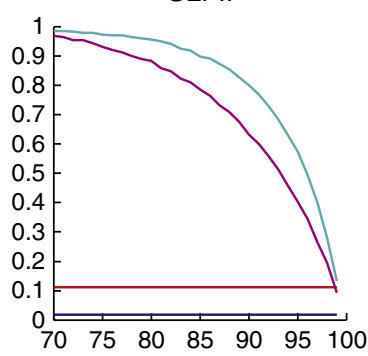

ILF

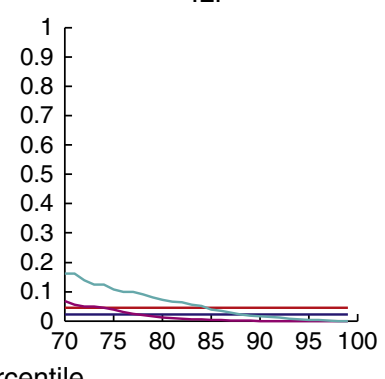

FP

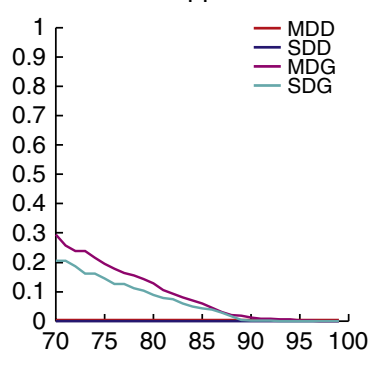

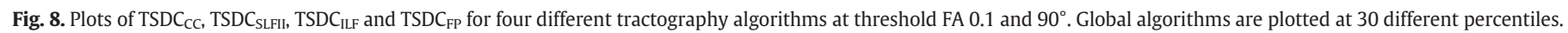

adjacency matrices at any threshold for these cases (see Fig. 1, upper row, second panel), we are justified in quantitatively comparing the SDG and MDG connections for the same percentiles (van Wijk et al., 2010). It can be seen that at 90th and 95th percentile thresholds most of the expected transcallosal inter-hemispheric projections are correctly identified with very few false positives with both methods. One can observe a greater tendency in SDG for false negatives at the green arrows compared to MDG in the 99th percentile and slightly more false positives for SDG in the white circle in the 95th percentile. In summary, the greater sensitivity and more complete inference of the transcallosal projections by MDG quantified by the TSDC $\mathrm{CC}_{\mathrm{CC}}$ is achieved without a compromise in false negatives as observed in the tractography paths and in the specificity TSDC in the 95th and 99th percentiles.

To assess the effect on connection topology in more spatial detail, Fig. 11 shows the spatial distribution of degrees for nodes on the cortical surface for both MDG and SDG for the same thresholds as Fig. 10. The effect of probabilistic threshold is to sharply decrease the absolute number of edges for each node and to focus the remaining edges more on the most highly connecting hubs. Spatial degree distributions can also be seen to be more focused for SDG than for MDG. Specifically, connection hubs identified by both approaches in the same location (for instance the lateral inferior frontal hub) are more spatially confined and have a higher absolute degree for SDG. However, MDG defines some hubs, such as the lateral superior parietal area, that are much less clear in the SDG.

Next, we look at the second branch of the superior longitudinal fasciculus (SLFII), which is a slightly shorter associative tract characterized by a high curvature and fewer tracts crossing it. Again Fig. 8 shows TSDC $_{\text {SLFII }}$ to be larger for global tractography methods than for local approaches except for the 99th percentile compared to MDD. However, although MDD performs better than SDD, the $\mathrm{TSDC}_{\text {SLFII }}$ for SDG is larger than that for MDG for all probabilistic thresholds. Fig. 12 shows the corresponding tractography paths, at the 99th percentile for SDG and MDG, corresponding to the intersection of local and global TSDC SLFI. $_{\text {. It }}$ can be seen that at these high probabilistic thresholds the global algorithms preferentially connect the ROI patches that are spatially closest, a tendency that reduces with lower probabilistic percentiles (not shown for SLFII), which is also seen in the corresponding connected cortical patches reported in Supplementary Fig. S7. This can also be seen by a concentration of density in the corner of their adjacency submatrix. Instead patch connections for the local deterministic approaches, especially multi direction, are much more spatially spread over the ROIs. MDD is seen to detect more separated branches of the SLFII than SDD, SDG or MDG, which it is generally

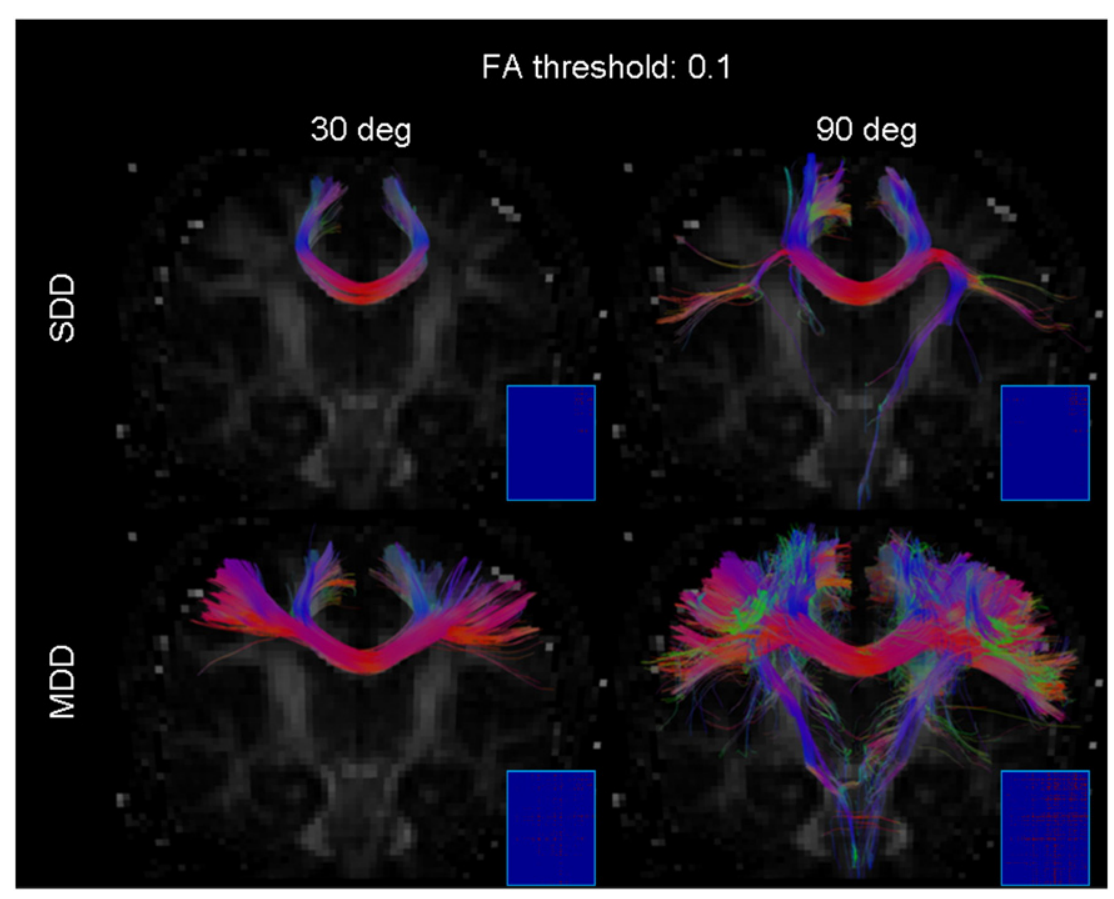

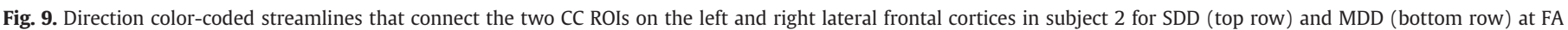
threshold 0.1 and two different angle thresholds. The corresponding submatrices obtained from the adjacency matrix (see Fig. 7) are shown for every combination. 


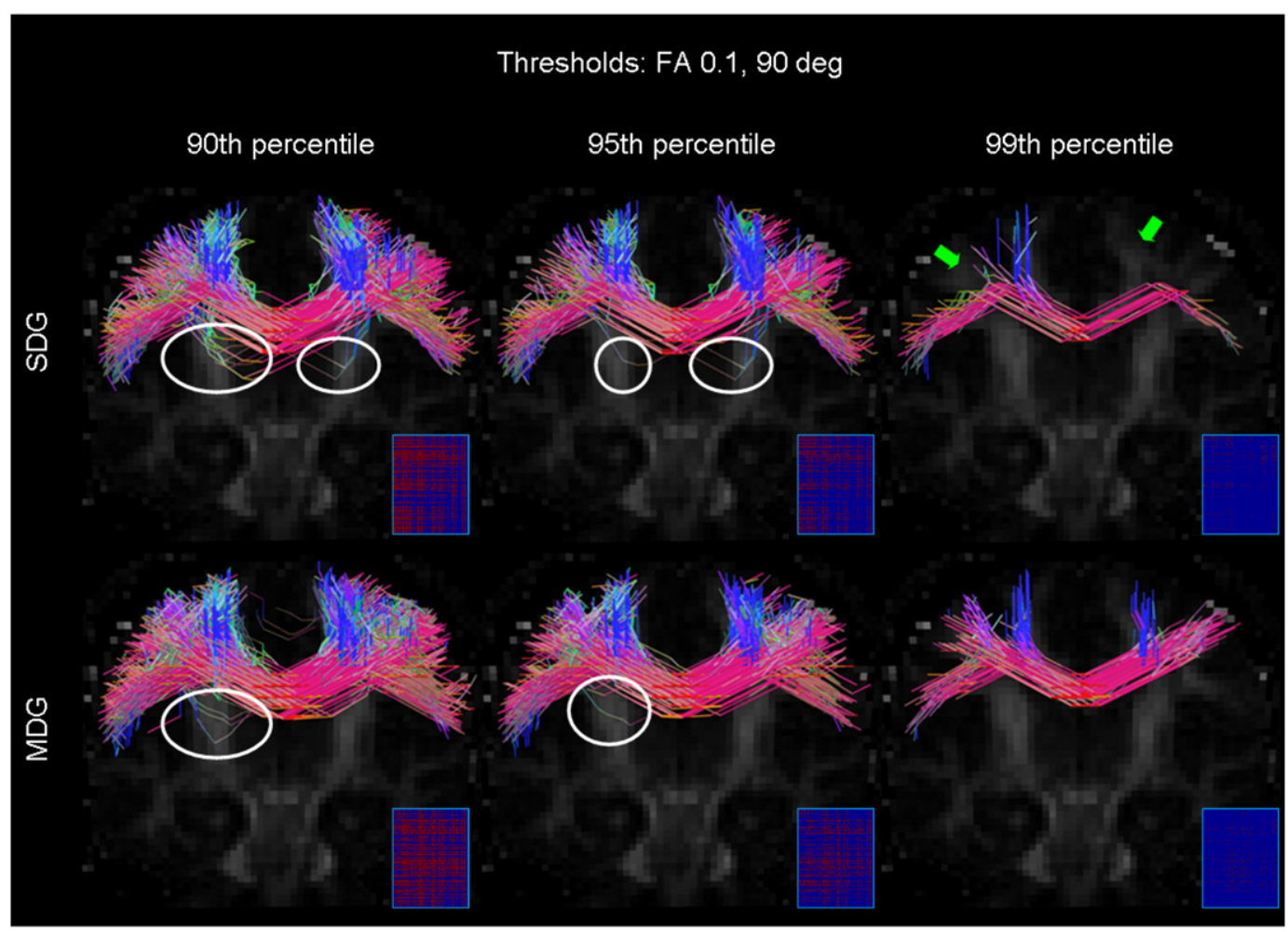

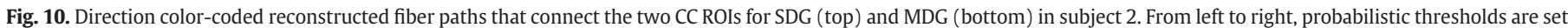

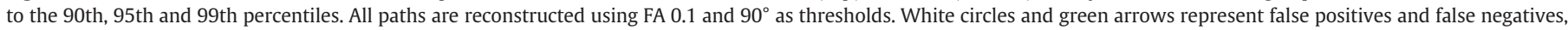
respectively. The corresponding submatrices obtained from the adjacency matrix (see Fig. 7) are shown for every combination.

considered to have (Ffytche et al., 2010; Thiebaut de Schotten et al., 2011), and to have a greater spread of connections in the most posterior regions. Furthermore, the superior performance of SDG and MDD in

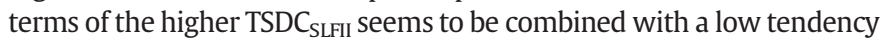
for false positives. Finally, MDG is seen to be more sensitive to noise and even more constrained in the length of the tract than SDG in this noncrossing tract.

Finally we have selected the left ILF of subject 2 to show aberrant behavior that can occur even in data with good SNR when identification of local direction is imperfect. The ILF connects the anterior part of the temporal lobe to the occipital lobe. It is characterized by a very straight course along the temporal lobe and it is joined in its path by several other fibers, such as the inferior fronto-occipital fasciculus (IFO). In particular the arcuate fasciculus (AF) curves down from its course from the perisylvian cortex of the frontal and parietal lobes and converges to join the direction of the ILF (Catani and Thiebaut de Schotten, 2008). This convergence of directions of two major tracts within the imaging voxels creates particularly difficult local direction identification problem. This is because crossing fibers are harder to identify for multiple direction models as the different directions become more colinear. As can be seen in Fig. 13 with the corresponding connected cortical patches reported in Supplementary Fig. S8, the ILF is well identified by all methods. However, Fig. 8 shows TSDC $\mathrm{ILF}_{\mathrm{ILF}}$ to be two to ten times lower than that for the other two tracts for all methods except for SDD.

Table 2

The absolute number of streamlines and average streamline lengths for each of the tractography results in Fig. 7

\begin{tabular}{lcc}
\hline Tractography method & Number of streamlines & $\begin{array}{l}\text { Average streamline } \\
\text { length [mm] }\end{array}$ \\
\hline SDD, $30^{\circ}$ & 3325 & 108.26 \\
SDD, $90^{\circ}$ & 11,981 & 113.70 \\
MDD, 30 & 10,703 & 92.03 \\
MDD, $90^{\circ}$ & 49,237 & 118.23 \\
\hline
\end{tabular}

Furthermore, the TSDC ILF for SDG and MDG shows a much steeper decline with an increasing probabilistic threshold. More careful inspection of Fig. 13 shows the reason to be a relatively long tract in conjunction with imperfect local direction segregation highlighted by the zoomed inset. This shows MDD to take unrealistically nearly $90^{\circ}$ turns which seem to result from jumping out of the ILF into the AF and then converging back into the ILF again. MDG in contrast avoids tracing through this region altogether. This is because MDG's global shortest path finding mechanism penalizes such strong local curvature for the entire tract, which for the relatively long ILF is already penalized for its length in global probabilistic approaches.

\section{Discussion}

We have investigated how different tractography algorithms can influence the estimation of several network indices that characterize the human structural connectome, including small-worldness, average clustering coefficient, characteristic path length, global network efficiency and high density nodes. We show that the choice of the general class of tractography algorithm in combination with the thresholds on FA, angle and probabilistic percentiles can dramatically influence these network indices. This is true in many cases even when global density differences are controlled for, showing that this marks actual differences in network topology.

It is important to note that the findings of small-worldness, high global network efficiency and high density hubs per se, characteristic for the human brain (Hagmann et al., 2008), are very robust over all investigated methods and parameters. These findings are also very consistent over two subjects and over different random surface parcellations for any of the tested combinations. It is rather the relative strength of expression of these effects that is found to be affected by model, algorithm and parameter choices. This is important because there is limited consensus on what, even within our general classes methods, the reference method for structural connectome tractography should be. Previous 


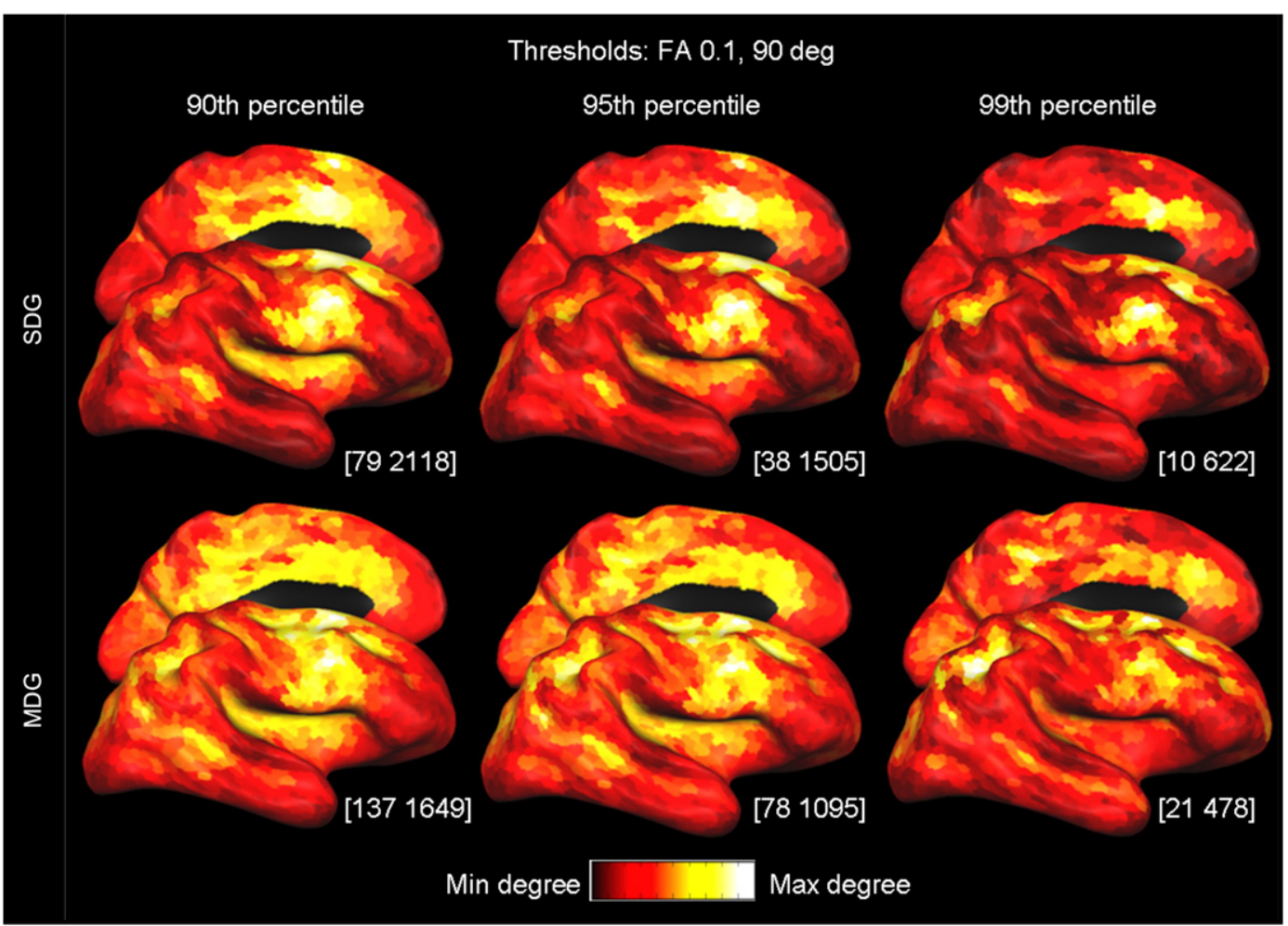

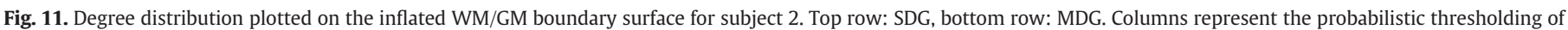
the connectivity matrix for the 90th, 95th and 99th percentiles. Between brackets are the min-max degree intervals for each combination.

work has used methods in a large subset of our general classes, including MDD (e.g. Hagmann et al., 2008; Zalesky et al., 2010), SDD (e.g. Zalesky et al., 2010), MDP (e.g. Li et al., 2011), SDG (e.g. Iturria-Medina et al., 2007) and MDG (e.g. Iturria-Medina et al., 2011). There are many intra-voxel diffusion models and tractography algorithms that could be used besides the ones we have chosen here. We listed some of the available alternatives in Table 1 and in the Introduction section. However, rather than performing an exhaustive unstructured test of all or most available methods, we have chosen to examine the effect of three common distinctions that almost any approach is characterized by. These are the distinctions between i) single fiber direction versus multiple directions in the intra-voxel diffusion model, ii) deterministic versus probabilistic tractography and iii) local versus global measure-of-fit tractography. Within each class defined by these distinctions (SDD, MDD, SDP, MDP, SDG and MDG) we chose one of the most used or most common methods as the reference. Obviously, other choices could have been made here. For instance in the SDD class, variants of the deterministic streamline algorithm exist which use different local direction finding (such as tensor deflection or TEND), step integration approaches (e.g. 4 th order Runge-Kutta) and ODF interpolation techniques. DSI, QBI, and the ball-and-stick model are all quite commonly used as multidirection models for tractography and PROBTRACKX (Behrens et al., 2007 ) is an often used alternative for iFOD for local probabilistic multi-direction tractography. Of course, the particular strategy selected from each general class might not be representative of the class in general in all its varieties and detail. However, for the level of general comparison that we aim at here, the subtle distinctions between methods within a general class listed in Table 1 is not the focus of investigation. In addition, a practical constraint is formed by the need to create a whole brain connection matrix, requiring many thousands of runs of any tractography algorithm, which is computationally challenging especially for probabilistic and global tractography methods.

The effect of algorithm and parameter choice has two components. First, there is the large effect of tractography algorithm and parameters on global network density, which is known to strongly affect graph indices. We have found that connection density generally decreases: i) with increasing probabilistic threshold, ii) when going from probabilistic to deterministic tractography, iii) with increasing FA and decreasing angle thresholds, iv) when moving from global to local tractography, and v) when moving from multi direction to single direction models. Second, more importantly, there are remaining effects on graph indices even when global density is controlled for which range in the tens of percent. It is important to note that maximizing absolute small-worldness is never the aim in quantifying this measure in any given individual or in a comparison. Instead, the aim is to have a robust, specific and sensitive measure of global connection topology in the brain as a useful dependent variable.

A pertinent question that has been addressed, at least in part, in a number of previous studies - though not always in the context of connectome reconstruction - is what reasons we have to choose multiple direction models over single, probabilistic over deterministic and global over local tractography. Given the effect on topology measures discussed above, the question of the optimal tractography approach becomes even more important. Therefore, in the second part of this paper we have presented an approach to quality control part of the connectome adjacency matrices which is suitable for comparing the different tractography approaches. Conceivably, it could be used to quality control entire connectome matrices by requiring sensitivity TSDCs in a certain range for a considerable number of known tracts and specificity TSDCs to be low for a number of known not-to-be-connected ROIs, which together span a large fraction of the adjacency matrix. This approach uses connectome dissection into a few large associative bundles which are known to exist and calculation of quantitative TSDCs. This is combined with careful inspection of tractography paths themselves to allow for assessment of true/false positives and true/false negatives. This brings us beyond the mere assessment of global measures, such as graph density or average streamline length, to the actual connections in the brain that are reconstructed by more streamlines or longer streamlines. We can use these results to inform choices in the tractography algorithms. Below we discuss the magnitude and direction 


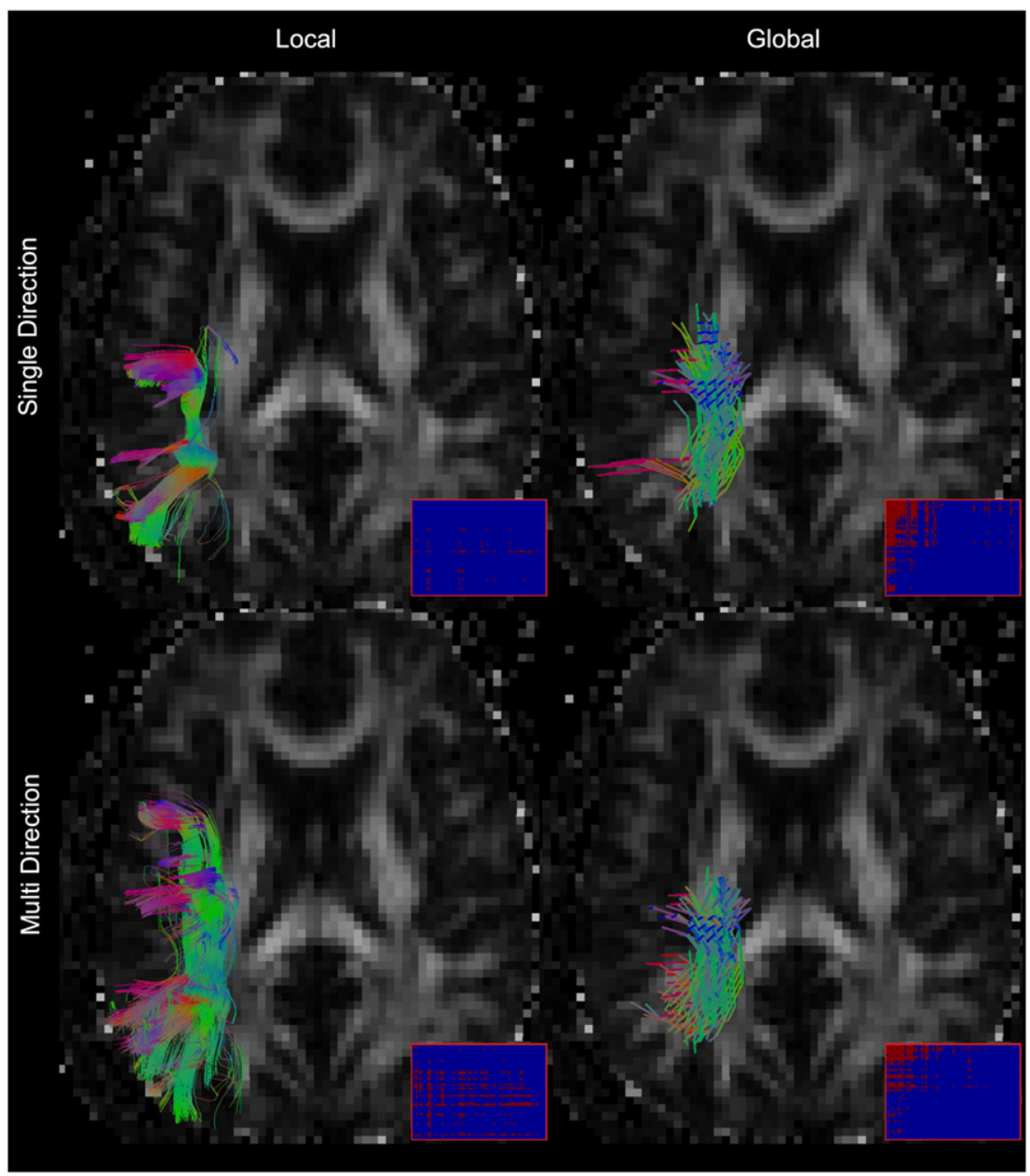

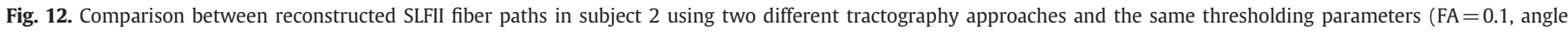

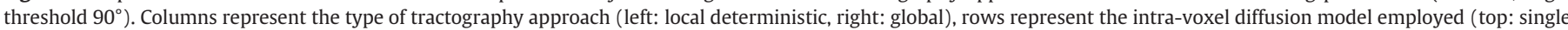

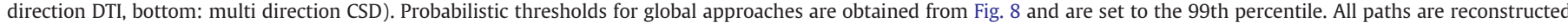
using FA 0.1 and $90^{\circ}$ as thresholds. The corresponding submatrices obtained from the thresholded adjacency matrix are shown for every combination.

of the effects of these choices on global connection topology measures, how these choices interact with each other and with threshold parameters and the signal-to-noise ratio (SNR) of the DWI data, and which choice is favored by the proposed quality control approach.

\section{Single versus multiple directions}

The main effect of moving from single direction intra-voxel diffusion models to multi-direction models is an increase in the number of long streamlines or tractography paths (see Fig. 2 and Table 2 for deterministic algorithms), which is further increased by liberal angle thresholds. Thus, multi-direction models yield higher density and global network efficiency and lower small-worldness than single-direction ones at the same percentile threshold (see Supplementary Figs. 1-5). This agrees with other reports of greater network connection density for deterministic tractography on multiple direction models compared to the DTI model (Bassett et al., 2011a; Zalesky et al., 2010). For global approaches, the spatial degree distribution is less focused on high density nodes for MDG, perhaps because longer tractography paths are retained compared to SDG (Fig. 11). When identifying exclusively topological differences by matching graph densities, small-worldness is seen to be larger under some circumstances for multiple directions than single direction, which is explained by a smaller normalized path length, with normalized average clustering coefficient being relatively unaffected.

The connectome dissection $\mathrm{QC}$ is most decisive on the choice of single versus multiple directions in the case of the inter-hemispheric projections through the body of the corpus callosum. In agreement with earlier reports (Behrens et al., 2007; Tuch et al., 2003; Wedeen et al., 2008), this shows a preference for multiple direction models over single direction ones for tractography. This is motivated by higher $\mathrm{TSDC}_{\mathrm{CC}}$ and more veridical results for multiple direction models, along with a low number of false positives for appropriate probabilistic thresholds as quantified by the TSDC $C_{\mathrm{FP}}$. Thus, this is an interesting example of a justifiable method choice that has the effect of decreasing the expression of high density nodes, since at least part of the increased long range density is formed by true connections that were otherwise missed.

A general point in fitting models to data is clearly exposed in comparing multiple versus single direction models, namely that of model complexity and sensitivity to noise, or of bias and variance. A multi direction model is more sensitive to the SNR of a diffusion weighted dataset and will show greater variability in reconstructed fiber orientations as SNR becomes lower because more parameters must be estimated. This can result in spurious (false positives) or missing (false negatives) fiber directions identified in single noisy voxels. The simpler single direction models such as DTI are more robust against noise having much fewer parameters to identify which can work very well in areas with simple fiber architectures such as the SLFI. This might represent a constraint when deciding which model to use for the analysis of diffusion weighted data. When performing a case-control study, for example, a simple single direction DTI-based SDD approach can be preferable compared to an MDD one, when it reduces the inter-subject variability within groups 


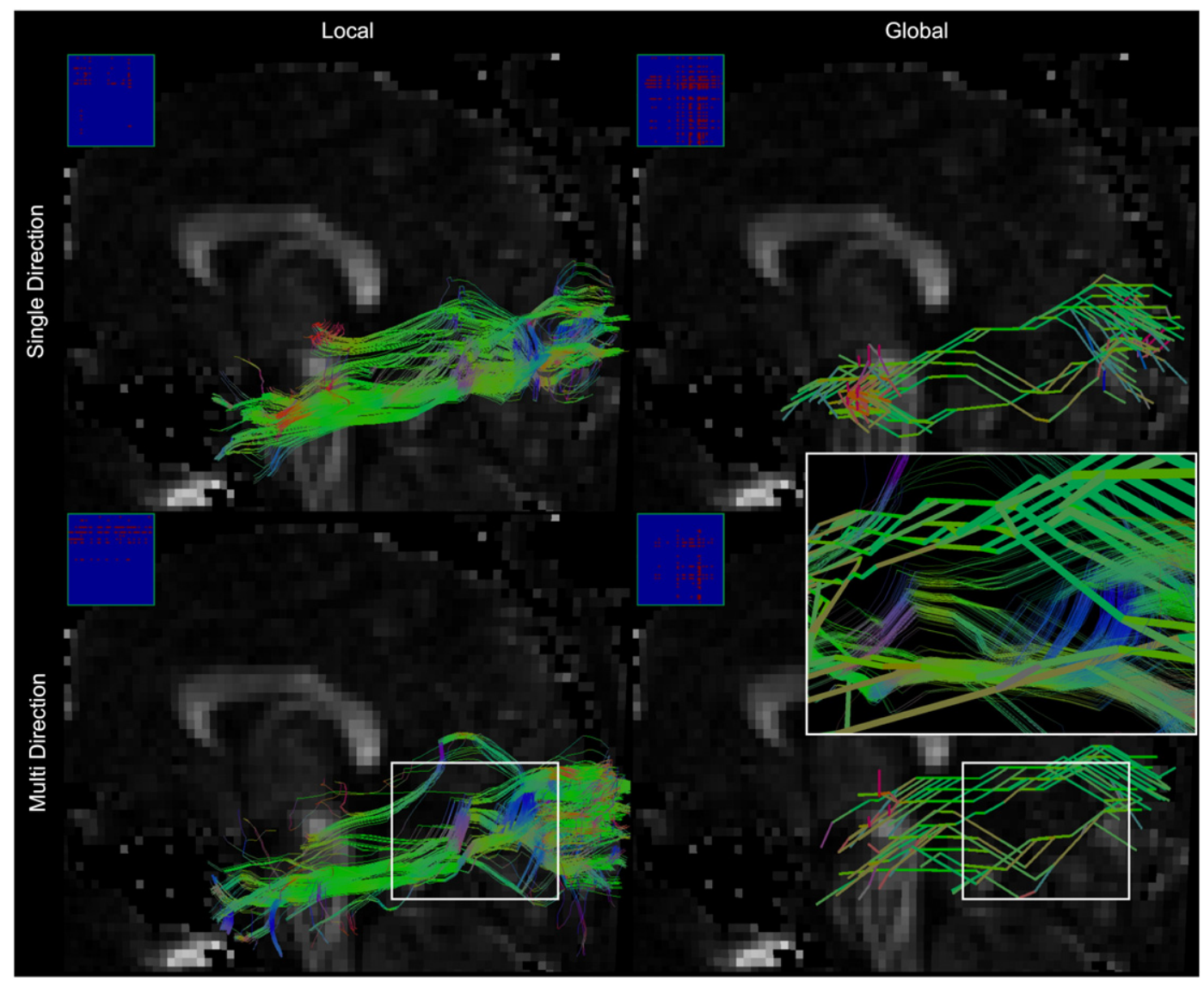

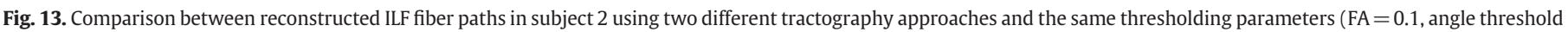

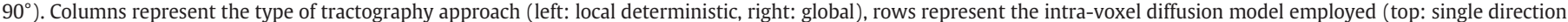

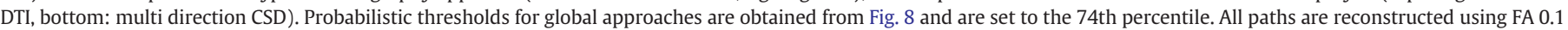

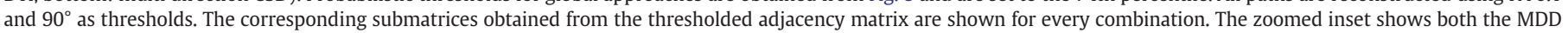
streamlines as thin lines and the MDG fiber paths as thick lines.

explaining more variance at an acceptable cost of delivering more false negatives.

\section{Deterministic versus probabilistic}

With increasing probabilistic threshold adjacency matrix density decreases, as one would expect when eliminating edges by thresholding on probabilistic weights. Generally, this decrease in density is accompanied by higher small-worldness (mostly through increase of normalized averaged clustering coefficient) and lower global network efficiency (see Supplementary Figs. 1-5). Furthermore, for the global algorithms employed here, a higher probabilistic threshold increased the focus of high density nodes, i.e. it increased the sharpness of peaks in the degree distribution over the cortical nodes (Fig. 11). Since probabilistic weights fall off with path length and as evidenced by a higher clustering (a higher relative abundance of local connections), it follows that increasing probabilistic thresholds prunes the network to lower density mostly by removing long range connections.

When holding all other parameters constant, deterministic methods generally capture all the extremes of the effects that were observed for increasing probabilistic threshold. In other words, a 'deterministic limit of probabilistic results' is observed experimentally in the case of edge density and can be considered to form a $100 \%$ limit value for the entire probabilistic threshold curves. Note that this is not meant to imply that the high threshold limit of probabilistic tractography results is necessarily equivalent to that of a deterministic algorithm seeded in the same location. The high threshold limit value result for deterministic approaches here only applies to global network characteristics derived from whole brain tractography. When looking at pure topological properties of the network by matching the densities, deterministic algorithms have much larger small-worldness than probabilistic algorithms which is mainly explained by a much shorter characteristic path length. This is true both for a seeding strategy for deterministic algorithms in the entire WM (diamonds in Figs. 3-6) and only at the WMbound (squares in Figs. 3-6). Normalized average clustering coefficient is less affected by this choice, although differences up to $10 \%$ can be observed.

The conceptual advantage of probabilistic tractography is that it quantifies both average pathways and variation in tractography results (Behrens et al., 2003; Parker et al., 2003), which is desirable. However, in the context of connectome reconstruction a practical problem is to choose a probabilistic threshold which - as we show here - has great influence on global network statistics. This problem has briefly been touched upon before without extensive investigation by other authors. In Hagmann et al. (2008) and Zalesky et al. (2010) connectome reconstruction was performed with deterministic tractography and an inter-node weight calculation that involves a number of connecting streamlines, node area volume and/or surface and streamline lengths, as was done here for SDD and MDD. In Li et al. (2011), a single probabilistic threshold is chosen by matching the resulting adjacency matrix density to earlier work (Achard and Bullmore, 2007). Finally, some investigations of adjacency matrices derived from both functional MRI data and DWI tractography have employed integration of results over a smaller or larger range of threshold values on fMRI correlations (from 50th to 99.9th percentiles; Achard and Bullmore, 2007) or 
probabilistic tractography percentile (from 99th to 99.9th percentiles; Gong et al., 2009). This reveals that either by deterministic fiber reconstruction or by selection of relatively high probabilistic thresholds most work has strived for sparse networks (low density) along the continuum sketched in Fig. 1.

An absolute probabilistic threshold independent of a desired final density level is difficult to set since probabilistic tractography visitation counts or weights are hard to interpret in an absolute way (Jbabdi and Johansen-Berg, 2011; Jones, 2008a, 2010). First, probabilistic weights for streamlines or paths fall off naturally with path length, which in a large part explains the longer characteristic path length compared to density matched adjacency matrices obtained by deterministic algorithms. Second, a probabilistic weight quantifies a mixture of noise in the data (affecting precision of the result), bias in the employed model and the true likelihood of a connection (affecting accuracy of the result). Subsets of these challenges have started to be addressed by Monte Carlo or bootstrap approaches (Jeurissen et al., 2011; Jones, 2008b; Morris et al., 2008). However, these methods are computationally very expensive, even for a single seeding point or ROI, and applying them to connectome reconstruction provides a tremendous computational challenge. Interestingly, the connectome dissection QC approach outlined here offers a quantitative way to define relatively narrow range of tolerable probabilistic threshold for connectome tractography. This is the range of values which simultaneously offers good sensitivity and veridicality of connections (sensitivity TSDCs between 0.1 and 0.5 ) and good specificity (specificity TSDCs below 0.01). In the example of the transcallosal projections this leads to a tolerable range between the 91st and the 98th percentiles, which agrees to good qualitative delineation of these connections (see Fig. 10).

In addition to the effect of probabilistic threshold on tractography path length, there is also an effect of different tractography seeding strategies. Seeding from all of white matter is biased toward longer fiber bundles because they are sampled with a greater number of seed points, whereas seeding from the boundary is biased toward recovering shorter bundles (Li et al., 2011). We have equalized this seeding bias between tractography algorithms by using the same boundary seeding strategy for all methods (squares in Figs. 3-6 for SDD and MDD).

Again there are considerations of model complexity and sensitivity to noise. Local probabilistic methods at very liberal thresholds, especially a 90 degree angle threshold in absence of a colinearity constraint based on prior identification of main diffusion directions can lead to degeneration of algorithm performance and pathological results. This is understandable, given that less restrictive thresholds (FA and angle) decrease modeling assumptions and resulting biases (such as: 'low curvature is good') but also increase variability in the results through the effects of noise. As discussed above, this effect is more pronounced for more complex models with more parameters, such as multi direction models compared to single direction models (Fig. 9). Pathological noise-dominated results can occur when this complexity and lack of constraints is then combined with the controlled variability introduced at each step by local probabilistic algorithms. Among the range of combination of methods and parameters settings, the combination of local probabilistic algorithms with a $90^{\circ}$ angle threshold without a colinearity constraint between fiber direction and local diffusion direction (essentially a low-curvature bias) is certainly an extreme one which is rarely employed in practice. However, it is important to point out that the main reason to use a limited maximum angle (minimum curvature) is not necessarily biologically inspired: nerve bundles and white matter fascicles sometimes can turn sharply (e.g. Roebroeck et al., 2008). Rather, it is the regularizing and smoothing effect of the low-curvature assumption that helps in avoiding sensitivity to excessive noise effects. Therefore, it is interesting to construct and evaluate tractography algorithms that achieve a high robustness to noise with fewer constraints.

\section{Local versus global}

Going from a local to a global approach affects the estimation of the reconstructed fiber paths in two important ways. First, global methods lead to a higher connection density than their local probabilistic counterparts at the same percentile thresholds (see Fig. 1), likely due to a higher number of longer connections. This means that to match density between global and local probabilistic algorithms implies applying a relatively high percentile threshold to the global and a relatively low one to the local. Nevertheless, when density matched, local shows higher small-worldness than global explained in a large part by a shorter characteristic path length. This is because all long tractography paths have been suppressed in the global results because of the comparatively high threshold. Second, in the connectome dissection QC, the sensitivity/specificity trade-off of the reconstructed fiber paths is qualitatively better for global methods than for the local deterministic ones with the same parameters. For instance, in Figs. 9 and 10 global methods allow robust tractography even for very liberal thresholds, such as a $90^{\circ}$ angle threshold, which is reflected in a high TSDC $\mathrm{CC}$ in conjunction with high specificity (low $\mathrm{TSDC}_{\mathrm{FP}}$ ) for appropriate probabilistic thresholds. This decrease in vulnerability to local noise is actually the motivating property of global tractography methods (Iturria-Medina et al., 2007; Jbabdi et al., 2007; Sherbondy et al., 2009; Sotiropoulos et al., 2010). In addition, the weighted shortest-path finding approach still discourages very long-range connecting fibers (as local probabilistic methods do) which is often a biologically plausible constraint in the brain that can avoid false positives. See, for example, the false positive connections between left and right lateral frontal cortices via the brainstem in SDD and MDD in Fig. 9 that are absent for SDG and MDG in Fig. 10. All in all, global algorithms show higher TSDCs implying higher sensitivity over large ranges of percentile thresholds, as shown in the TSDC $_{\mathrm{CC}}$ and TSDC $\mathrm{SLFII}_{\text {. }}$ Crucially, this is in conjunction with high specificity (very low $\mathrm{TSDC}_{\mathrm{FP}}$ ) for probabilistic threshold between approximately the 91st and the 98th percentiles. It is only when comparing to deterministic tractography at very high thresholds (which match TSDCs between methods) that their short path bias weights in strongly as seen for instance in Fig. 12. Here it is important to note that when not trying to match density or TSDC to a deterministic algorithm, there is no a-priori reason to apply such high threshold to global probabilistic results. Finally, the example of the ILF shows interesting differences in behavior between SDD, MDD and global algorithms. In this case, where identification of local direction is imperfect, TSDC for SDD is the same as for the other tracts, TSDC for MDD is reduced by a factor 2 to a factor 3 and TSDC for SDG and MDG is decreased by an order of magnitude. This shows that for a combination of irregularities in the data and a long tract, global algorithms can show a decrease in performances which is, however, picked up very well by the connectome dissection QC. Although the performance of SDD is very consistent across different tracts, it is important to remark that it shows a consistently low sensitivity. For instance, the TSDC $C_{\text {SLFII }}$ and TSDC $_{\text {ILF }}$ are as low as for the clearly underconnected CC (see Fig. 9), despite specificity for SLFII (see Fig. 12) and ILF (see Fig. 13) being rather good.

Two disadvantages of global methods must be mentioned. First, there is their high computational cost. Probabilistic methods, global even more than local approaches, were the most time consuming methods to run for the very large whole-brain tractography sets. In addition, this computational cost will increase more than linearly with increase in spatial resolution of the DWI data as MR hardware and sequences advance (e.g. Feinberg et al., 2010; Heidemann et al., 2010). Second, to keep computational complexity within reasonable bounds (in the limit: to avoid trying to exhaustively cover an infinity of options), global algorithms need constraints on the allowable shapes of pathways. This can be achieved either by discretizing a parameterization (the so-called Hough-transform) of continuous curves through the data (such as polynomials of a given order; Aganj et al., 2011) or by discretizing the allowable curves on a 3D grid as in the 
graph-based tractography algorithms employed here. As a consequence, from a local point of view, both the step size and the angle of local curvature in moving from one gridpoint to the next are constrained and anisotropic. That is, in the $5 \times 5 \times 5$ local neighborhood used here there are 98 discrete unique directions to move in with a few different step sizes. It is important to note that this is indeed a very 'local streamline' way of looking at a global algorithm, since local possibly erroraccumulating steps are never actually performed in graph tractography. Rather, the complete enumeration of all possible discrete traversals through the entire dataset is searched. In this search for a shortest weighted path the local weights are defined by the DWI data modeling using the entire solid angle around a step direction such that any directional peak in the data is taken into account (Iturria-Medina et al., 2007; Sotiropoulos et al., 2010). Nevertheless, it can be appreciated in Figs. 10, 12 and 13 that this approach will lead to fiber pathways that are more discrete in nature. Thus, given the combined advantages of multidirection models and global tractography, investigation into computationally feasible global approaches with a minimum of pathway shape constraints may prove useful for whole brain structural network reconstruction studies.

\section{Conclusion}

We have shown that general choices in tractography algorithm in combination with the employed thresholds on FA, angle and probabilistic percentiles have a large effect on derived human structural brain network indices. This effect has two components. First, there is the large effect of tractography algorithm and parameters on global network density, which is known to strongly affect graph indices. Second, more importantly, there are remaining effects even when global density is controlled for. Although small-worldness, global network efficiency and density are a robust finding over all investigated methods and parameters, their absolute strength of expression can be changed by several tens of percent, which is significant in their role as a sensitive and accurate metric to distinguish subtle differences in global network topology. Since maximizing the absolute value of network indices is not the aim in itself, the reasons we have to choose multiple fiber direction models over single direction models, probabilistic over deterministic and global over local tractography methods are more subtle.

We investigate this with the proposed connectome dissection QC in which evaluation of TSDCs measure sensitivity and specificity in combination with careful inspection of tractography path results. Conceivably, it could be used within a semi-automated quality control framework for entire connectome matrices by requiring sensitivity TSDCs in a certain range for a considerable number of known tracts and specificity TSDCs to be low for a number of known not-to-be-connected ROIs, which together span a large fraction of the adjacency matrix. This would require development or application of an atlas of such end-ROI which can be mapped to individual datasets and maturation of conventions for tolerable TSDC value limits, which is an important topic of future work.

Here, the results on sensitivity and specificity for a subset of tractography paths corroborate earlier investigations that conclude a superiority of multiple direction models for intra-voxel fiber direction modeling. More tentatively, probabilistic tractography might be preferred over deterministic for its capacity to quantify the variability of tracking results. However, the choice of probabilistic threshold in the context of whole brain structural network delineation still remains an open issue of importance. Interestingly, the connectome dissection QC approach outlined here offers a quantitative way to define relatively narrow range of tolerable probabilistic threshold for connectome tractography. This is the range of values which simultaneously offers good sensitivity and veridicality of connections (sensitivity TSDCs between 0.1 and 0.5 ) and good specificity (specificity TSDCs below 0.01). Fig. 8 nicely shows that in this way TSDCs can quantify sensitivity
(CC and SLFII), lack of sensitivity (ILF) and specificity (FP). Probabilistic local tractography algorithms can exhibit considerable noise sensitivity when applied to multi direction models without strong constraints on local tract curvature. Therefore, global approaches that exhibit less sensitivity to local noise may be preferred, particularly when more of the available SNR is used in the DWI acquisition to improve spatial resolution. Development of global tractography methods that balance reasonable computational demands with acceptable constraints aimed at limiting the search space (such as discretization) might further improve their applicability.

\section{Acknowledgments}

The authors would like to thank Alexandros Goulas, Federico de Martino, Kâmil Uludağ and Yasser Iturria-Medina for helpful comments and suggestions on earlier versions of this manuscript. They would also like to thank two reviewers for valuable suggestions which have significantly improved this manuscript. This work was funded by EU Marie Curie Initial Training Network Grant PITN-GA-2009-238593.

\section{Appendix A}

\section{Parcellation scheme}

The equal-area surface parcellation procedure used in this work is based on constrained centroidal Voronoi tessellations (Du et al., 2003). This approach relies on the iterative Lloyd's algorithm, which is often used in computer science to produce point distributions over two or three-dimensional surfaces with blue noise characteristics. A blue noise distribution ensures uniformity and regularity in the sampling without showing any bias in the Fourier spectrum.

The proposed algorithm uses Voronoi patches to sub-divide the initial surface into $n$ different ROIs. By definition, a Voronoi patch comprises all the points which are closest to its centroid. Initially, a Voronoi tessellation is used whose centroids are randomly defined (white noise distribution) over the two dimensional surface. Iteratively, the algorithm shifts the centroid of each cell to its barycentre by minimizing the following equation:

$\min _{z \in S} \int_{V} \rho(x)|x-z|^{2} d x$

where $z$ is the new centroid, $x$ are the points comprised in a certain Voronoi patch, $S$ is the surface, $V$ is the Voronoi patch and $\rho$ is a density function, here uniformly set to 1 . Note that, since finding the exact barycenter on a 2D surface based on geodesic distances is an NP hard problem, in the proposed algorithm it is always confined to be one of the vertices in the patch and, therefore, trivially lies on the 2D surface. This approach, being more computationally tractable, uses an approximate estimate of the real barycenter. At every step, the algorithm computes the geodesic distances between all the surface points and the neighbors of the current barycenter of a certain patch. The new barycenter is the neighbor point which minimizes the proposed equation.

The distances between the centroids and the other points are not 3D Euclidean but geodesics along the 2D surface. The algorithm converges to make the areas of the patches as close to each other as possible.

\section{Appendix B. Supplementary data}

Supplementary data to this article can be found online at http:// dx.doi.org/10.1016/j.neuroimage.2012.06.002. 


\section{References}

Achard, S., Bullmore, E., 2007. Efficiency and cost of economical brain functional networks. PLoS Comput. Biol. 3, e17.

Aganj, I., Lenglet, C., Jahanshad, N., Yacoub, E., Harel, N., Thompson, P.M., Sapiro, G., 2011. A Hough transform global probabilistic approach to multiple-subject diffusion MRI tractography. Med. Image Anal. 15, 414-425.

Assaf, Y., Basser, P.J., 2005. Composite hindered and restricted model of diffusion (CHARMED) MR imaging of the human brain. Neurolmage 27, 48-58.

Basser, P.J., Pierpaoli, C., 1996. Microstructural and physiological features of tissues elucidated by quantitative-diffusion-tensor MRI. J. Magn. Reson. B 111, 209-219.

Basser, P.J., Mattiello, J., LeBihan, D., 1994. MR diffusion tensor spectroscopy and imaging. Biophys. J. 66, 259-267.

Basser, P.J., Pajevic, S., Pierpaoli, C., Duda, J., Aldroubi, A., 2000. In vivo fiber tractography using DT-MRI data. Magn. Reson. Med. 44, 625-632.

Bassett, D.S., Brown, J.A., Deshpande, V., Carlson, J.M., Grafton, S.T., 2011a. Conserved and variable architecture of human white matter connectivity. Neurolmage 54, 1262-1279.

Bassett, D.S., Wymbs, N.F., Porter, M.A., Mucha, P.J., Carlson, J.M., Grafton, S.T., 2011b. Dynamic reconfiguration of human brain networks during learning. Proc. Natl. Acad. Sci. U. S. A. 108, 7641-7646.

Behrens, T.E., Woolrich, M.W., Jenkinson, M., Johansen-Berg, H., Nunes, R.G., Clare, S. Matthews, P.M., Brady, J.M., Smith, S.M., 2003. Characterization and propagation of uncertainty in diffusion-weighted MR imaging. Magn. Reson. Med. 50, 1077-1088.

Behrens, T.E., Berg, H.J., Jbabdi, S., Rushworth, M.F., Woolrich, M.W., 2007. Probabilistic diffusion tractography with multiple fibre orientations: what can we gain? Neurolmage 34, 144-155.

Cammoun, L., Gigandet, X., Meskaldji, D., Thiran, J.P., Sporns, O., Do, K.Q., Maeder, P., Meuli, R., Hagmann, P., 2012. Mapping the human connectome at multiple scales with diffusion spectrum MRI. J. Neurosci. Methods 203, 386-397.

Catani, M., Thiebaut de Schotten, M., 2008. A diffusion tensor imaging tractography atlas for virtual in vivo dissections. Cortex 44, 1105-1132.

Cheng, H., Wang, Y., Sheng, J., Sporns, O., Kronenberger, W.G., Mathews, V.P., Hummer, T.A., Saykin, A.J., 2011. Optimization of seed density in DTI tractography for structural networks. J. Neurosci. Methods 203 (1), 264-272.

Conturo, T.E., Lori, N.F., Cull, T.S., Akbudak, E., Snyder, A.Z., Shimony, J.S., McKinstry, R.C., Burton, H., Raichle, M.E., 1999. Tracking neuronal fiber pathways in the living human brain. Proc. Natl. Acad. Sci. U. S. A. 96, 10422-10427.

Cook, P.A., Bai, Y., Nedjati-Gilani, S., Seunarine, K.K., Hall, M.G., Parker, G.J., Alexander, D.C., 2006. Camino: open-source diffusion-MRI reconstruction and processing. 14th Scientific Meeting of the International Society for Magnetic Resonance in Medicine, Seattle, WA, USA.

Du, Q., Gunzburger, M.D., Ju, L.L., 2003. Constrained centroidal Voronoi tessellations for surfaces. SIAM J. Sci. Comput. 24, 1488-1506.

Feinberg, D.A., Moeller, S., Smith, S.M., Auerbach, E., Ramanna, S., Gunther, M., Glasser, M.F., Miller, K.L., Ugurbil, K., Yacoub, E., 2010. Multiplexed echo planar imaging for sub-second whole brain FMRI and fast diffusion imaging. PLoS One 5, e15710.

Ffytche, D.H., Blom, J.D., Catani, M., 2010. Disorders of visual perception. J. Neurol. Neurosurg. Psychiatry 81, 1280-1287.

Fillard, P., Descoteaux, M., Goh, A., Gouttard, S., Jeurissen, B., Malcolm, J., RamirezManzanares, A., Reisert, M., Sakaie, K., Tensaouti, F., Yo, T., Mangin, J.F., Poupon, C., 2011. Quantitative evaluation of 10 tractography algorithms on a realistic diffusion MR phantom. NeuroImage 56, 220-234.

Frank, L.R., 2001. Anisotropy in high angular resolution diffusion-weighted MRI. Magn. Reson. Med. 45, 935-939.

Goebel, R., Esposito, F., Formisano, E., 2006. Analysis of functional image analysis contest (FIAC) data with BrainVoyager QX: from single-subject to cortically aligned group general linear model analysis and self-organizing group independent component analysis. Hum. Brain Mapp. 27, 392-401.

Gong, G., Rosa-Neto, P., Carbonell, F., Chen, Z.J., He, Y., Evans, A.C., 2009. Age- and genderrelated differences in the cortical anatomical network. J. Neurosci. 29, 15684-15693.

Hagmann, P., Kurant, M., Gigandet, X., Thiran, P., Wedeen, V.J., Meuli, R., Thiran, J.P., 2007. Mapping human whole-brain structural networks with diffusion MRI. PLoS One 2, e597.

Hagmann, P., Cammoun, L., Gigandet, X., Meuli, R., Honey, C.J., Wedeen, V.J., Sporns, O., 2008. Mapping the structural core of human cerebral cortex. PLoS Biol. 6, e159.

Heidemann, R.M., Porter, D.A., Anwander, A., Feiweier, T., Heberlein, K., Knosche, T.R Turner, R., 2010. Diffusion imaging in humans at 7T using readout-segmented EPI and GRAPPA. Magn. Reson. Med. 64, 9-14.

Iturria-Medina, Y., Canales-Rodriguez, E.J., Melie-Garcia, L., Valdes-Hernandez, P.A., Martinez-Montes, E., Aleman-Gomez, Y., Sanchez-Bornot, J.M., 2007. Characterizing brain anatomical connections using diffusion weighted MRI and graph theory. NeuroImage 36, 645-660.

Iturria-Medina, Y., Perez Fernandez, A., Morris, D.M., Canales-Rodriguez, E.J., Haroon, H.A., Garcia Penton, L., Augath, M., Galan Garcia, L., Logothetis, N., Parker, G.J., Melie-Garcia, L., 2011. Brain hemispheric structural efficiency and interconnectivity rightward asymmetry in human and nonhuman primates. Cereb. Cortex 21, 56-67.

Jarbo, K., Verstynen, T., Schneider, W., 2012. In vivo quantification of global connectivity in the human corpus callosum. NeuroImage 59, 1988-1996.

Jbabdi, S., Johansen-Berg, H., 2011. Tractography: where do we go from here? Brain Connect. 1, 169-183.

Jbabdi, S., Woolrich, M.W., Andersson, J.L., Behrens, T.E., 2007. A Bayesian framework for global tractography. NeuroImage 37, 116-129.

Jeurissen, B., Leemans, A., Jones, D.K., Tournier, J.D., Sijbers, J., 2011. Probabilistic fiber tracking using the residual bootstrap with constrained spherical deconvolution. Hum. Brain Mapp. 32, 461-479.
Jones, D.K., 2008a. Studying connections in the living human brain with diffusion MRI. Cortex 44, 936-952.

Jones, D.K., 2008b. Tractography gone wild: probabilistic fibre tracking using the wild bootstrap with diffusion tensor MRI. IEEE Trans. Med. Imaging 27, 1268-1274.

Jones, D.K., 2010. Challenges and limitations of quantifying brain connectivity in vivo with diffusion MRI. Imaging Med. 2, 341-355.

Lazar, M., Weinstein, D.M., Tsuruda, J.S., Hasan, K.M., Arfanakis, K., Meyerand, M.E. Badie, B., Rowley, H.A., Haughton, V., Field, A., Alexander, A.L., 2003. White matter tractography using diffusion tensor deflection. Hum. Brain Mapp. 18, 306-321

Leemans, A., Jones, D.K., 2009. The B-matrix must be rotated when correcting for subject motion in DTI data. Magn. Reson. Med. 61, 1336-1349.

Li, L., Rilling, J.K., Preuss, T.M., Glasser, M.F., Hu, X., 2011. The effects of connection reconstruction method on the interregional connectivity of brain networks via diffusion tractography. Hum. Brain Mapp. (Electronic publication ahead of print) http:// dx.doi.org/10.1002/hbm.21332.

Liu, C., Bammer, R., Acar, B., Moseley, M.E., 2004. Characterizing non-Gaussian diffusion by using generalized diffusion tensors. Magn. Reson. Med. 51, 924-937.

Mori, S., Crain, B.J., Chacko, V.P., van Zijl, P.C., 1999. Three-dimensional tracking of axonal projections in the brain by magnetic resonance imaging. Ann. Neurol. 45, 265-269.

Morris, D.M., Embleton, K.V., Parker, G.J., 2008. Probabilistic fibre tracking: differentiation of connections from chance events. Neurolmage 42, 1329-1339.

Ozarslan, E., Mareci, T.H., 2003. Generalized diffusion tensor imaging and analytical relationships between diffusion tensor imaging and high angular resolution diffusion imaging. Magn. Reson. Med. 50, 955-965.

Ozarslan, E., Shepherd, T.M., Vemuri, B.C., Blackband, S.J., Mareci, T.H., 2006. Resolution of complex tissue microarchitecture using the diffusion orientation transform (DOT). NeuroImage 31, 1086-1103.

Parker, G.J., Alexander, D.C., 2005. Probabilistic anatomical connectivity derived from the microscopic persistent angular structure of cerebral tissue. Philos. Trans. R Soc. Lond. B Biol. Sci. 360, 893-902.

Parker, G.J., Haroon, H.A., Wheeler-Kingshott, C.A., 2003. A framework for a streamlinebased probabilistic index of connectivity (PICo) using a structural interpretation of MRI diffusion measurements. J. Magn. Reson. Imaging 18, 242-254.

Ramayya, A.G., Glasser, M.F., Rilling, J.K., 2010. A DTI investigation of neural substrates supporting tool use. Cereb. Cortex 20, 507-516.

Roebroeck, A., Galuske, R., Formisano, E., Chiry, O., Bratzke, H., Ronen, I., Kim, D.S. Goebel, R., 2008. High-resolution diffusion tensor imaging and tractography of the human optic chiasm at 9.4 T. NeuroImage 39, 157-168.

Rubinov, M., Sporns, O., 2010. Complex network measures of brain connectivity: uses and interpretations. NeuroImage 52, 1059-1069.

Schultz, T., Seidel, H.P., 2008. Estimating crossing fibers: a tensor decomposition approach. IEEE Trans. Vis. Comput. Graph. 14, 1635-1642.

Sherbondy, A.J., Dougherty, R.F., Ben-Shachar, M., Napel, S., Wandell, B.A., 2008. ConTrack: finding the most likely pathways between brain regions using diffusion tractography. J. Vis. 8 (15), 11-16.

Sherbondy, A.J., Dougherty, R.F., Ananthanarayanan, R., Modha, D.S., Wandell, B.A 2009. Think global, act local; projectome estimation with BlueMatter. Med. Image Comput. Comput. Assist. Interv. 12, 861-868.

Sotiropoulos, S.N., Bai, L., Morgan, P.S., Constantinescu, C.S., Tench, C.R., 2010. Brain tractography using Q-ball imaging and graph theory: improved connectivities through fibre crossings via a model-based approach. NeuroImage 49, 2444-2456.

Sporns, O., Tononi, G., Kotter, R., 2005. The human connectome: a structural description of the human brain. PLoS Comput. Biol. 1, e42.

Thiebaut de Schotten, M., Dell'Acqua, F., Forkel, S.J., Simmons, A., Vergani, F., Murphy, D.G., Catani, M., 2011. A lateralized brain network for visuospatial attention. Nat. Neurosci. 14, 1245-1246.

Tournier, J.D., Calamante, F., Gadian, D.G., Connelly, A., 2004. Direct estimation of the fiber orientation density function from diffusion-weighted MRI data using spherical deconvolution. Neurolmage 23, 1176-1185.

Tournier, J.D., Calamante, F., Connelly, A., 2007. Robust determination of the fibre orientation distribution in diffusion MRI: non-negativity constrained super-resolved spherical deconvolution. NeuroImage 35, 1459-1472.

Tournier, J.D., Calamante, F., Connelly, A., 2010. Improved probabilistic streamlines tractography by 2 nd order integration over fibre orientation distributions. Proceedings of the Joint Annual Meeting ISMRM-ESMRMB 2010, Stockholm, Sweden.

Tuch, D.S., 2004. Q-ball imaging. Magn. Reson. Med. 52, 1358-1372.

Tuch, D.S., Reese, T.G., Wiegell, M.R., Makris, N., Belliveau, J.W., Wedeen, V.J., 2002. High angular resolution diffusion imaging reveals intravoxel white matter fiber heterogeneity. Magn. Reson. Med. 48, 577-582.

Tuch, D.S., Reese, T.G., Wiegell, M.R., Wedeen, V.J., 2003. Diffusion MRI of complex neural architecture. Neuron 40, 885-895.

van Wijk, B.C., Stam, C.J., Daffertshofer, A., 2010. Comparing brain networks of different size and connectivity density using graph theory. PLoS One 5, e13701.

Wakana, S., Jiang, H., Nagae-Poetscher, L.M., van Zijl, P.C., Mori, S., 2004. Fiber tractbased atlas of human white matter anatomy. Radiology 230, 77-87.

Watts, D.J., Strogatz, S.H., 1998. Collective dynamics of 'small-world' networks. Nature 393, 440-442.

Wedeen, V.J., Hagmann, P., Tseng, W.Y., Reese, T.G., Weisskoff, R.M., 2005. Mapping complex tissue architecture with diffusion spectrum magnetic resonance imaging. Magn. Reson. Med. 54, 1377-1386.

Wedeen, V.J., Wang, R.P., Schmahmann, J.D., Benner, T., Tseng, W.Y., Dai, G., Pandya, D.N Hagmann, P., D'Arceuil, H., de Crespigny, A.J., 2008. Diffusion spectrum magnetic resonance imaging (DSI) tractography of crossing fibers. Neurolmage 41, 1267-1277.

Westin, C.F., Maier, S.E., Mamata, H., Nabavi, A., Jolesz, F.A., Kikinis, R., 2002. Processing and visualization for diffusion tensor MRI. Med. Image Anal. 6, 93-108. 
Yeh, F.C., Wedeen, V.J., Tseng, W.Y., 2010. Generalized q-sampling imaging. IEEE Trans Med. Imaging 29, 1626-1635.

Zalesky, A., 2008. DT-MRI fiber tracking: a shortest paths approach. IEEE Trans. Med. Imaging 27, 1458-1471.

Zalesky, A., Fornito, A., 2009. A DTI-derived measure of cortico-cortical connectivity. IEEE Trans. Med. Imaging 28, 1023-1036.
Zalesky, A., Fornito, A., Harding, I.H., Cocchi, L., Yucel, M., Pantelis, C., Bullmore, E.T., 2010. Whole-brain anatomical networks: does the choice of nodes matter? NeuroImage 50, 970-983.

Zhang, Y., Brady, M., Smith, S., 2001. Segmentation of brain MR images through a hidden Markov random field model and the expectation-maximization algorithm. IEEE Trans. Med. Imaging 20, 45-57. 